\title{
Die Vorrechte des Adels in Bayern und Tirol. Steuer-, Gerichts- und Jagdprivileg im 16. und 17. Jahrhundert
}

\author{
Nikolaus Bliem \\ Kerngebiet: Neuzeit \\ eingereicht bei: Univ.-Ass. Dr. Niels Grüne M.A. \\ eingereicht im: WS 2013/14 \\ Rubrik: BA-Arbeit
}

\begin{abstract}
The Prerogatives of the Nobility in Bavaria and Tyrol. Tax, Jurisdiction and Hunting Privileges in the $16^{\text {th }}$ and $17^{\text {th }}$ Century

This bachelor thesis examines whether the nobility was a homogenous group in terms of privileges granted by the sovereign or not. It is argued that the amount and extent of these could vary. As shown for Bavaria and Tyrol and the selected privileges, the nobility was eager to preserve its claims while the sovereign and the bourgeoisie tried to diminish them. This developed into a continuous struggle of lower nobility clinging to their claims jealously. As a result the nobility was neither acting as one group nor were its privileges always accessible to them.
\end{abstract}

\section{Einleitung}

Adel als Herrschaftsträger gibtes nicht oder kaum mehr. In Europa ist der noch „,herrschende“ Adel zumeist auf symbolische Herrschaft beschränkt. In den konstitutionellen Monarchien Europas, von Großbritannien über die Niederlande bis hin zu Spanien ist der (Hoch-) Adel zwar weiterhin unnahbar, die wirkliche Macht liegt jedoch in den Parlamenten oder Regierungen. Heutzutage tritt der Adel häufiger in Boulevard- und „Klatschmagazinen“ auf als in der Politik und hat sich nur noch - so scheint es - den Prominentenstatus erhalten. Dabei hatte der Adel sehr lange eine überragende Stellung in der Gesellschaft eingenommen. Das Wort Adel kommt vom althochdeutschen adal und bedeutet Geschlecht oder Stammfolge. Der verwandte Begriff odal, sinngemäß Erbsitz bzw. ererbter Boden, lässt auch auf den im deutschsprachigen Raum notwendigen Besitz von Grund und Boden schließen, um dem Adelsstand anzugehören. Ein anderes Wort für Adel, 
Nobilität, leitet sich vom lateinischen nobiles ab, das Grundlage für die Bezeichnung des Adels in vielen anderen europäischen Sprachen ist. Dieses weist auf die für den Adel notwendige Distinktion von anderen hin, denn das Verb noscere, von dem nobiles abgeleitet ist, bedeutet kenntlich machen.'

In diesem Sinne der Distinktion nahm der Adel eine besondere Stellung für sich in Anspruch. Als Adeliger war man überlegen, und das sollte auch offensichtlich sein, nicht nur durch die Zurschaustellung von Reichtum, sondern vor allem aufgrund besonderer Rechte bzw. Vorrechte. Diese Privilegien konnten vielschichtig und sehr unterschiedlich sein und dienten der Aufgabe, sich nach unten abzugrenzen.

Walter Demel charakterisiert in seiner Einführung zur Geschichte des Adels sechs Gruppen von Privilegien: Erstens die sogenannten Ehrenvorrechte, was von einem besonderem Platz in der Kirche bis hin zum Recht Waffen zu tragen reichte. Als zweite Privilegiengruppe führt er die Rechte vor Gericht an; ein Adeliger durfte nur durch einen Adeligen gerichtet werden, darüberhinaus waren Strafen für den Adel meist weniger streng, sie waren "standesgemäßer". Ökonomische Exklusivrechte bzw. Monopolrechte sind als dritter Punkt zu nennen, wie etwa das Vorrecht des Adels in Russland auf das Brennen von Vodka. Hierzu zählt Demel aber auch das Recht, auf fremdem Grund und Boden jagen zu dürfen. Ein weiterer zentraler Punkt für den Adel waren seine politischen Privilegien. Der Adel stellte eine eigenständige Kurie auf Land- und Reichstagen dar und nahm Ämter und die Ausübung von Gerichtsbarkeit in Anspruch. In einem fünften Punkt nennt Demel die Befreiung von Abgaben oder Diensten, wie etwa Einquartierungen oder Milizdienst. Außerdem sei der Adel von Steuern befreit gewesen. Als abschließender Punkt wird das Recht des Adels angeführt, das Familienvermögen möglichst zusammenzuhalten. Der Adel hatte dementsprechend auch Vergünstigungen im Erbrecht. ${ }^{2}$

Rudolf Endres hebt in der Beschreibung der adeligen Vorrechte den besonderen Gerichtsstand, die niedere Gerichtsbarkeit (wozu häufig auch die Hochgerichtsbarkeit hinzugekommen sei), die Siegelmäßigkeit, die Nachlassinventur, die Fideikommisse, die unbeschränkte Lehensfähigkeit, die Ehrenvorrechte und die Sonderrechte bei Jagd-, Zoll-, Maut- und Steuerwesen hervor. ${ }^{3}$

Einen weiteren „Privilegienkatalog” stellt Michael Sikora in seiner Einführung Adel in der Frühen Neuzeit vor: Er versteht unter ökonomischen Vorrechten vor allem das Privileg, keine Steuern zahlen zu müssen, sowie Nutzungs- und Monopolrechte. Daneben verweist Sikora auf den besonderen Gerichtsstand und insbesondere auf die "Herrschaft über Land und Leute“ als zentrales Privileg des Adels, das von diesem in zwei Richtungen gerechtfertigt wurde: Einerseits durch ein Versprechen gegenüber dem Lehnsherren, inm im Kriegsfall beizustehen, andererseits den Untertanen Schutz zu bieten. ${ }^{4}$

1 Eckart Conze, Adel, in: Kleines Lexikon des Adels. Titel, Throne, Traditionen, hrsg. v. ders., München 2005, S. 15-18, hier S. 16.

2 Walter Demel, Der europäische Adel. Vom Mittelalter bis zur Gegenwart, München 2005, S. 54-56.

3 Rudolf Endres, Adel in der Frühen Neuzeit (Enzyklopädie Deutscher Geschichte 18), München 1993, S. 3 f.

4 Michael Sikora, Der Adel in der Frühen Neuzeit (Geschichte kompakt), Darmstadt 2009, S. 5 f. 
Trotz all dieser Privilegien, die den Adel sehr abgeschlossen und einheitlich erscheinen lassen, war der Adel, und dieser These folgt diese Arbeit, keineswegs eine homogene Gruppe, der jedes Privileg zustand, alleine weil sie diesem bevorzugten Stand angehörte, sondern unterschied sich teils stark. Vor allem der niedere Adel lässt sich als eine heterogene Gruppe charakterisieren, die sich ständig nach unten abzugrenzen versuchte und dies mit Hilfe von inm gewährten Privilegien absichern wollte. Gleichzeitig versuchte der Adel nach oben hin zum Landesherrn durch Erkämpfung dieser Privilegien so gut es ging aufzuschließen. Auch innerhalb des Adels ist eine Abgrenzung bzw. erwünschte Distinktion erkennbar. Der sogenannte neue Adel wurde innerhalb der Adelsgesellschaft nicht als vollwertig anerkannt. Die Heterogenität des niederen Adels ist daher weniger bei externen Einflüssen, wie unterschiedlichen Landesherrn oder der Zugehörigkeit zu unterschiedlichen Territorien zu suchen, sondern vielmehr in der unterschiedlichen Ausstattung mit Privilegien je nach Familie, je nach Grundherrschaft. Besaß eine Familie beispielsweise mehrere nicht reichsunmittelbare Gebiete (möglicherweise sogar unter verschiedenen Landesherrn), hatte sie darin nicht automatisch dieselben (Vor-)Rechte: So konnte ihr in einem Gebiet die hohe Gerichtsbarkeit zustehen, in einem anderen jedoch nur die niedere. Die Geschichte des niederen Adels ist daher ein ständiges Ringen um Vorrechte, um möglichst nahe an den hohen, herrschenden Adel heranzukommen und sich gleichzeitig innerhalb des Adels von den „Emporkömmlingen“ und außerhalb nach unten zum Nichtadel abzugrenzen, um sich so als besseren Stand zu positionieren.

Die Arbeit stellt sich daher folgenden Fragestellungen: Inwiefern standen dem Adel Privilegien offen? Gab es Unterschiede von Territorium zu Territorium? Gab es Konflikte aufgrund der adeligen Vorrechte, sei dies zwischen Landesherr und Adel, zwischen Adel und Untertanen oder innerhalb des Adels?

Der niedere Adel, der in dieser Arbeit im Fokus steht, unterscheidet sich vom Hochadel in erster Linie dadurch, dass er kein reichsunmittelbares Territorium besaß, d. h. dass zwischen dem niederen Adel und dem Kaiser noch ein Landesherr stand. ${ }^{5}$ Der Adel hatte im Alten Reich eine besondere Stellung inne, und als landsässiger Adel, also als Besitzer von Grund und Boden und als Grundherr, war er Mittelsmann zum Landesherrn, was den Alltag der Untertanen viel stärker bestimmte als die Oberherrschaft des Landesherrn oder gar des Kaisers. ${ }^{6}$

Der Adel wird häufig als eine homogene - vor allem homogen handelnde - Gruppe dargestellt und mit den Attributen Wohlstand und Macht versehen. Doch gerade die Konflikte des Adels über die Privilegien sowohl nach oben als auch nach unten sind für eine Untersuchung sehr interessant. Einerseits wurde damit eine Festigung der eigenen Vorrangstellung zur Distinktion von unteren Schichten versucht, andererseits nach weiterem Aufstieg gestrebt, um zusätzliche Befugnisse zu erlangen. In einem dritten Punkt sind die Konflikte innerhalb des vor allem niederen Adels bemerkenswert. So versuchte

5 Moritz Strachwitz, Niederer Adel, in: Kleines Lexikon des Adels. Titel, Throne, Traditionen, hrsg. v. Eckart Conze, München 2005, S. 187

6 Johannes Rogalla von Bieberstein, Adelsherrschaft und Adelskultur in Deutschland (Aus dem Deutschen Adelsarchiv 14), Limburg ${ }^{3}$ 1998, S. 4. 
der alte Adel, sich gegenüber „Emporkömmlingen“ aus dem Bürgertum zu wehren, die häufig eine Nobilitierung erlangt hatten und daher als Briefadel bezeichnet wurden.

Das ständige Ringen in diversen Konflikten wurde in der Forschung lange Zeit als Krise des Adels bezeichnet. Erst mit einem Perspektivenwechsel innerhalb der Forschung wurde dies als ein Wandel verstanden.

\section{Forschungsstand}

„Zentral aber ist für eine Gruppe wie den Adel als ganzes und für jedes einzelne Glied dieser Gruppe, deren zentrale Kategorie die Ausübung von Herrschaft und Beteiligung an Herrschaft ist, der Erhalt des adligen Status und womöglich seine Verbesserung und Hebung. Bedroht ist dieser Status immer" ${ }^{\prime 7}$.

Wie Peter Johanek im vorangestellten Zitat und in einem Artikel über den Adel in den österreichischen Gebieten herausstellt, wurdederAdelvondergeschichtswissenschaftlichen Forschung als sich in ständiger Krise befindend dargestellt und so ergebe sich das Bild, als ob sich diese Krise vom 13. bis zum 17. Jahrhundert durchziehe. Zudem findet sich in vielen Publikationen, maßgeblich der 1960 er- und 70er-Jahre, im Titel der Begriff "Krise“. ${ }^{8}$ Bekanntestes und viel zitiertes Beispiel dafür ist das 1965 erstmals erschienene Werk von Lawrence Stone The Crisis of the Aristocracy: 1558-1641.9

Johanek stellt dem entgegen, dass adlige Herrschaft und ihre ökonomische Basis, dass adliger Status sich insgesamt stets Bedrohungen ausgesetzt sieht, sich in einer prekären Situation befindet und durch wirtschaftliche wie politische Entwicklungen in solche Situationen gestellt wird, die Anpassungsprozesse erfordern, die bei erfolgreichem Ablauf den Status erhalten und bei Nichtgelingen dessen Verlust zur Folge haben können. ${ }^{10}$

Diese Erkenntnis schlug sich in der Folge in der Forschung nieder, und zwar in der Verwendung des Begriffes "Wandel“ anstatt „Krise“. ${ }^{11}$

Für diese Arbeit wurde wegen der vergleichenden Herangehensweise der beiden Untersuchungsräume Bayern und Tirol meist Forschungsliteratur herangezogen, die sich selbst auf eine der beiden Räume oder auf eine der untersuchten Privilegien beschränkt. Umfassende allgemeine Darstellungen der Privilegien sind selten und meist nur in sehr verkürzter Form in Einführungswerken zu finden, etwa in denen von Rudolf Endres, Walter Demel oder Michael Sikora. ${ }^{12}$

7 Peter Johanek, Der Adel in den österreichischen Ländern und in Tirol während des späten Mittelalters und der frühen Neuzeit, in: Die Wolkensteiner. Facetten des Tiroler Adels in Spätmittelalter und Neuzeit, hrsg. v. Kurt Andermann/Gustav Pfeifer (Veröffentlichungen des Südtiroler Landesarchivs 30), Innsbruck 2009 , S. 11-28, hier S. 14.

8 Johanek, Adel in den österreichischen Ländern und in Tirol, S 14.

9 Lawrence Stone, The Crisis of the Aristocracy: 1558-1641, Oxford 1965.

10 Johanek, Adel in den österreichischen Ländern und in Tirol, S. 14.

11 Ebd.

12 Demel, Der europäische Adel. Endres, Adel in der Frühen Neuzeit. Sikora, Der Adel in der Frühen Neuzeit. 
Im Bereich der Steuerfreiheiten des Adels ist in erster Linie auf zwei Abschlussarbeiten zu verweisen, die sich mit der Steuerpraxis in Teilen Tirols auseinandersetzen. Zum einen ist das die Dissertation von Barbara Painer, die die Stadt Meran als Untersuchungsgegenstand gewählt hat. ${ }^{13}$ Zum anderen hat Stefanie von Wenzl eine gute Diplomarbeit zu den Auseinandersetzungen des Adels mit der Stadt Bruneck im Hinblick auf die Steuern geschrieben. ${ }^{14}$ Erwähnenswert erscheint auch ein Artikel von Marcello Bonazza mit dem Titel Der Adel im Trentino und in Tirol und das Steuerwesen. ${ }^{15}$

Für Bayern ist einerseits die Monographie von Katrin Ellen Kummer von großer Bedeutung ${ }^{16}$, andererseits der Beitrag von Reinhard Heydenreuter im Sammelband Adel und Adelskultur in Bayern, herausgegeben von Walter Demel und Ferdinand Kramer. ${ }^{17}$ Dieser Artikel vermittelt zudem im Bereich der bayerischen Gerichtsbarkeit entscheidende Erkenntnisse. Einen eher allgemeinen Überblick zur Steuerpraxis in der Frühen Neuzeit gibt dagegen Martin Körner. ${ }^{18}$

Neben Heydenreuters bereits erwähntem Artikel stechen für die Gerichtsbarkeit in Bayern die Arbeit von Monika Ruth Franz zu den Landesordnungen von 1516 und $1520^{19}$ sowie die Artikel von Christian Wieland und Klaus Andrian-Werburg hervor. Für den Tiroler Raum nimmt die Habilitation von Martin Paul Schennach eine überragende Stellung ein. Schennach beschäftigt sich darin mit der Entstehung des Gesetzgebungsstaates der Frühen Neuzeit in Tirol. Auf beeindruckende Weise legt er darin bis ins kleinste Detail die Praxis der Gerichtsbarkeit dar, weswegen seine Arbeit einen sehr wichtigen Beitrag zum heutigen Stand der Forschung leistet. ${ }^{20}$

Eine ähnlich zentrale Arbeit hat Schennach auch zum Jagdrecht verfasst unter dem Titel Jagdrecht, Wilderei und "gute Policey".21 Ebenfalls zu erwähnen ist hierbei die ausführliche, aber etwas ältere Darstellung von Heinrich Oberrauch. ${ }^{22}$

13 Barbara Painer, Geschichte des Steuerwesens der Stadt Meran von 1438-1780, Innsbruck Diss. O. J.

14 Stephanie von Wenzl, Stadt und Adel in Bruneck. Auseinandersetzungen in der 2. Hälfte des 16. Jahrhunderts, Innsbruck Dipl. 2006.

15 Marcello Bonazza, La nobiltà trentino-tirolese di fronte al prelievo fiscale tra politica di ceto e comporrtamenti individuali, in: Geschichte und Region 4 (1995), S. 77-112.

16 Katrin Ellen Kummer, Landstände und Landschaftsverordnung unter Maximilian I. von Bayern (1598-1651) (Schriften zur Verfassungsgeschichte 74), Berlin 2005.

17 Reinhard Heydenreuter, Zur Rechtstellung des landsässigen Adels im Kurfürstentum Bayern zwischen dem 16. und 18. Jahrhundert, in: Adel und Adelskultur in Bayern, hrsg. v. Walter Demel/Ferdinand Kramer (Zeitschrift für bayerische Landesgeschichte Beiheft 32), München 2008, S. 43-105.

18 Martin Körner, Steuern und Abgaben in Theorie und Praxis im Mittelalter und in der frühen Neuzeit, in: Steuern, Abgaben und Dienste vom Mittelalter bis zur Gegenwart. Referate der 15. Arbeitstagung der Gesellschaft für Sozial- und Wirtschaftsgeschichte vom 14. bis 17. April 1993 in Bamberg, hrsg. v. Eckart Schremmer (Vierteljahresschrift für Sozial- und Wirtschaftsgeschichte Beiheft 114), Stuttgart 1994, S. 5376.

19 Monika Ruth Franz, Die Landesordnung von 1516/1520. Landesherrliche Gesetzgebung im Herzogtum Bayern in der ersten Hälfte des 16. Jahrhunderts (Bayerische Rechtsquellen 5), München 2003.

20 Martin P. Schennach, Gesetz und Herrschaft. Die Entstehung des Gesetzgebungsstaates am Beispiel Tirols (Forschungen zur deutschen Rechtsgeschichte 28), Köln-Weimar-Wien 2010.

21 Martin P. Schennach, Jagdrecht, Wilderei und "gute Policey“. Normen und ihre Durchsetzung im frühneuzeitlichen Tirol (Studien zu Policey und Policeywissenschaft), Frankfurt a.M. 2007.

22 Heinrich Oberrauch, Tirols Wald und Waidwerk. Ein Beitrag zur Forst- und Jagdgeschichte (SchlernSchriften 88), Innsbruck 1952. 
Allgemeinere Werke bieten Hans Wilhelm Eckardt 1976 und Werner Rösener 2004, wobei sich Rösener zu großem Teil auf Eckardts Erkenntnisse stützt. ${ }^{23}$ Trotz der auffälligen sprachlichen sowie stilistischen Ähnlichkeit zu Eckardt, die sich streckenweise nur durch minimale Veränderung des Satzbaus äußert, hat Rösener eine sehr umfassende Gesamtdarstellung der Geschichte der Jagd präsentiert, während Eckardt sich vornehmlich auf die sozialen Konflikte beschränkt, die mit dem Jagdrecht in der Frühen Neuzeit entstanden sind.

Abschließend ist noch auf diejenigen Publikationen hinzuweisen, die einen allgemeineren Charakter besitzen und daher Rückschlüsse für mehrere Abschnitte zuließen: Margit Ksoll hat eine umfassende Darstellung der adeligen Ökonomie vorgelegt, die nicht nur steuerliche Themen anspricht, sondern auch Gerichtsbarkeit und Jagdangelegenheiten. ${ }^{24}$ Der Artikel von Klaus Bleeck und Jörn Garber zu den Privilegien in Adelstheorien wird in dieser Arbeit zwar nicht ausdrücklich erwähnt, direkt oder indirekt zitiert, doch hat die Lektüre des Textes Denkanstöße vorangetrieben, die zur Entstehung dieser Arbeit beigetragen haben. ${ }^{25}$

Weiters sollen noch die Darstellungen zum österreichischen und Tiroler Adel von Peter Johanek wie auch der relevante Abschnitt im Handbuch der Geschichte des Landes Tirol und das kleine Heftchen von Viktor Ernst zur Entstehung und Charakteristik des niederen Adels nicht unerwähnt bleiben. ${ }^{26}$

\section{Aufbau und Konzept}

In der Beantwortung der Forschungsfragen und der gestellten These bedarf es einer Auseinandersetzung mit den beiden gewählten Untersuchungsräumen: Das Herzogtum Bayern und die Grafschaft Tirol. Während Bayern beispielsweise von dem 1525 ausgebrochenen Bauernkrieg großteils verschont blieb, trat in Tirol mit Michael Gaismair eine der zentralen Personen dieses Massenaufstandes der unteren Schichten auf.

Aus den in der Einleitung bereits kurz vorgestellten Privilegien, die dem Adel zugeschrieben werden, wurden für diese Arbeit drei ausgewählt, um in diesem überschaubaren Rahmen einige Ergebnisse präsentieren zu können:

Die Steuerfreiheit des Adels, die der Adel für sich beanspruchte, bezogen auf Abgaben jeglicher Art, ist das erste Privileg. Dem Landesherrn Geld abzugeben, empfand der Adel als seiner Würde und seinem Stande nicht entsprechend.

23 Hans Wilhelm Eckardt, Herrschaftliche Jagd, bäuerliche Not und bürgerliche Kritik. Zur Geschichte der fürstlichen und adligen Jagdprivilegien vornehmlich im südwestdeutschen Raum (Veröffentlichungen des Max-Planck-Instituts für Geschichte 48), Göttingen 1976. Werner Rösener, Die Geschichte der Jagd. Kultur, Gesellschaft und Jagdwesen im Wandel der Zeit, Düsseldorf-Zürich 2004.

24 Margit Ksoll, Die wirtschaftlichen Verhältnisse des bayerischen Adels 160-1679. Dargestellt an den Familien Törring-Jettenbach, Törring zum Stain sowie Haslang zu Haslangkriet und Haslang zu Hohenkammer (Schriftenreihe zur bayerischen Landesgeschichte 83), München 1986.

25 Klaus Bleeck/Jörn Garber, Nobilitas: Standes- und Privilegienlegitimation in deutschen Adelstheorien des 16. und 17. Jahrhunderts, in: Daphnis 11 (1982), H. 1-2, S. 49-114.

26 Johanek, Adel in den österreichischen Ländern und in Tirol. Rudolf Palme, Frühe Neuzeit (1490 - 1665), in: Geschichte des Landes Tirol, Bd. 2, hrsg. v. Josef Fontana u.a., Bozen 1986. Viktor Ernst, Die Entstehung des niederen Adels, Aalen 1965. 
Die Gerichtsbarkeit, die der Adel ausübte, und seine Privilegien in der Rechtsprechung stellen einen zweiten Bereich an Vorrechten dar, der in dieser Arbeit besprochen wird. Dem Adel war zumeist nicht nur eine besondere Behandlung vor Gericht zugesichert, sondern er trat häufig als Inhaber der Gerichtsbarkeit auf, auch der niedere Adel. Dieses Anrecht zeigte sich im Süden des Reiches in den so genannten Hofmarken.

Als dritter, abschließender Abschnitt werden die mit der Gerichtsbarkeit häufig, aber nicht zwingend, einhergehenden Jagdrechte behandelt. Dem Adel lag viel an dem Recht, jagen zu dürfen, und es war eines der am meisten gehegten Vorrechte, das aber auch zu vielen Konflikten führte, sowohl nach oben als auch nach unten.

Jedes dieser drei Privilegien wird zunächst kurz allgemein vorgestellt, dann getrennt für Bayern und Tirol betrachtet, anschließend wird ein kurzer Vergleich versucht.

\section{Untersuchungsräume}

Für diese Arbeit wurden zwei Territorien zur Betrachtung ausgewählt: das Herzogtum Bayern und die Grafschaft Tirol in dem Untersuchungszeitraum des gesamten 16. und des beginnenden 17. Jahrhunderts.

Ein Unterschied zwischen beiden Territorien sticht auf den ersten Blick heraus: In Bayern war der Adel sehr dominant, in Tirol spielte er keine derart wichtige Rolle. In Tirol waren Bauern im Allgemeinen freier und Grundherrschaft bzw. Leibeigentum nicht so stark ausgebildet wie in Bayern. Außerdem trat in Bayern ein Phänomen in der Gerichtsbarkeit auf, das es zwar auch in Tirol gab, jedoch nicht in dieser Bedeutung und starken Ausprägung: die Hofmark.

Im beginnenden 16. Jahrhundert regte sich im Alten Reich Unmut der unteren Schichten, der sich allmählich in hauptsächlich gegen den Adel gerichteten Unruhen und Aufständen zu entladen begann. Als Beispiele wären hier zu nennen: der Arme Konrad 1514 im Herzogtum Württemberg, die Bundschuh-Bewegungen in Südwestdeutschland Anfang des 16. Jahrhunderts. Eine Entwicklung, die im Bauernkrieg von 1525 gipfelte.

Von Schwaben ausgehend, erfasste dieser Aufstand einen Großteil des südlichen deutschsprachigen Raumes, unter anderem auch Tirol. Bayern blieb davon verschont, während ringsum die Bauern aufbegehrten, vor allem gegen die Privilegien des Adels. So sollte der Adel vor Gericht nicht mehr privilegiert sein und auch seine Gerichtsbarkeit bzw. seine Herrschaftsrechte sollten stark beschnitten werden, wie etwa in Tirol durch die Landesordnung von Michael Gaismair. Zusätzlich sollte der Adel „mitleiden“, wie die Bauern und auch die wirtschaftlichen Privilegien verlieren. Gemeint war damit auch maßgeblich das Steuerprivileg. ${ }^{27}$

Arbeiten, die regionalen Charakter aufweisen, sind häufig dem Vorwurf ausgesetzt, dass sie untereinander nicht zu vergleichen seien, da der gemeinsame Nenner fehle und

27 Horst Buszello, Legitimation, Verlaufsformen und Ziele, in: Der deutsche Bauernkrieg, hrsg. v. Peter Blickle/ Horst Buszello/Rudolf Endres, Paderborn u. a. ${ }^{3}$ 1995, S. 281-321, hier S. 297 f. 
sie keine einheitlichen Methoden und Herangehensweisen verwendeten. ${ }^{28}$ Dennoch stellt eine regionale Geschichtsschreibung zunehmend umfassende Möglichkeiten zum gegenseitigen Vergleich dar, woraus dann möglicherweise ein Blick auf das Ganze gewagt werden kann. Für diese Arbeit hat sich der regionale Charakter alleine aufgrund des Umfanges zwingend ergeben, wobei der Versuch einer Gesamtdarstellung der adeligen Privilegien geradezu anmaßend wäre und ohne jeglichen Anspruch auf Allgemeingültigkeit auskommen müsste.

\section{Untersuchungsraum Bayern}

Zu Beginn des 16. Jahrhunderts veränderte sich Bayern grundlegend: Seit dem 14. Jahrhundert war Bayern erbgeteilt und wurde erst durch einen Schiedsrichterspruch Maximilians des I. 1505, der den Landshuter Erbfolgekrieg beendete, wiedervereinigt, verlor aber dabei die Gerichte Kufstein, Rattenberg, Kitzbühel sowie das Zillertal an die Grafschaft Tirol, da Maximilian diese aufgrund seiner Tätigkeit als Schiedsrichter beanspruchte. Bayern bestand daraufhin aus vier Rentmeisterämtern, die von München aus regiert wurden: München, Landshut, Straubing und Burghausen. ${ }^{29}$

Damit Bayern nicht erneut geteilt werden konnte, wurde 1506 nach Zustimmung der Landstände die Primogenitur beschlossen, wonach der Erstgeborene männliche Nachkomme des Herzogs allein erbberechtigt war. ${ }^{30}$

Die Regierung der wittelsbachischen Herzöge hob sich durch ihr starkes Durchsetzungsvermögen hervor. So gab es in ihrem Einflussbereich vergleichsweise sehr wenige reichsunmittelbare adelige Herrschaften. Die Landesfürsten griffen sehr stark in die Rechte des landsässigen Adels ein und ließen inm kaum Eigenständigkeit. ${ }^{31}$

Daher ist im 16. Jahrhundert in Bayern auch eine immer kleiner und gleichmäßiger werdende Adelsschicht zu bemerken. So waren von den 630 landsässigen Familien Mitte des 15. Jahrhunderts bis zum Jahr 1557 über 300 ausgestorben oder aus anderen Gründen nicht mehr von Bedeutung. ${ }^{32}$ Zudem gab es in Bayern keinen zahlenmäßig starken armen Landadel. Besitz konzentrierte sich nicht in dem Ausmaß wie dies etwa beim böhmischen Adel der Fall war. ${ }^{33}$

Angesichts der vergleichsweise schwachen Position des Adels hatte er verhältnismäßig starkes Gewicht in der Landschaft, wo er die Hälfte aller Sitze innehatte und an der Wende zur Neuzeit noch eine sehr starke Rolle in der Politik spielte. Im Laufe des 16. Jahrhunderts kann von einem Machtverlust des niederen Adels in Bayern gesprochen werden, bei

28 Joachim Schneider, Spätmittelalterlicher deutscher Niederadel. Ein landschaftlicher Vergleich (Monographien zur Geschichte des Mittelalters 52), Stuttgart 2003, S. 13.

29 Franz, Die Landesordnung von 1516/1520, S. 40*.

30 Ebd., S. 41 *.

31 Heydenreuter, Rechtstellung des landsässigen Adels, S. 46.

32 Klaus Freiherr von Andrian-Werburg, Der altbaierische Adel im landesfürstlichen Staat der Wittelsbacher bis zum Abschluss der ritterschaftlichen Verfassung, in: Deutscher Adel. 1430-1555. Büdinger Vorträge 1963 hrsg. v. Helmuth Rössler (Schriften zur Problematik der deutschen Führungsschichten in der Neuzeit 1), Darmstadt 1965, S. 48-57, hier S. 55.

33 Bieberstein, Adelsherrschaft und Adelskultur, S. 72. 
gleichzeitiger Machtkonzentration auf den Landesfürsten. Daher ist hier auch von einer, im Vergleich zu anderen Territorien, raschen und sehr erfolgreichen Staatswerdung zu sprechen, auch wenn die erneut für den niederen, alten Adel bekräftigten Privilegien Mitte des 16. Jahrhunderts (wie später noch ausgeführt werden wird) für den Landesherren kontraproduktiv erscheinen. ${ }^{34}$

Wie unten erläutert werden wird, gab es wie in Tirol auch „in“ Bayern kleinere Territorien zumeist geistlicher Natur, die reichsunmittelbar waren. Diese gehörten zwar formal und rechtlich nicht zu Bayern, waren jedoch aufs Engste mit dem Herzogtum verbunden. Viele dieser Gebiete konnten sich die Wittelsbacher durch eine aggressive Politik einverleiben. Einige wenige konnten sich jedoch halten. Ein Beispiel dafür ist das Hochstift Freising. Etwas nördlich von München gelegen, hatte das Hochstift ein geistliches Oberhaupt, das mit Herrschaftsrechten versehen war. Durch die enge Verbundenheit, die zweifellos bestand, waren Grenzen nie genau festgelegt und bis ins 16. Jahrhundert auch nicht als wichtig empfunden worden. Erst durch die fortschreitende Institutionalisierung bemühte sich der bayerische Landesherr um eine genaue Klärung der Kompetenzen. Vor allem ging es dabei um Zuständigkeiten im Strafrecht, also in der Gerichtsbarkeit. Diese Politik wurde wie zuvor bei den weltlichen Herrschaften sehr aggressiv geführt und führte zu endlosen Auseinandersetzungen mit dem Stift. ${ }^{35}$

\section{Untersuchungsraum Tirol}

Zum Raum der Grafschaft Tirol gehörten rechtlich die reichsunmittelbaren Bistümer Brixen und Trient nicht. Ihre Geschichte ist aber, so wie in Bayern, mit derjenigen der Grafschaft Tirols aufs Engste verbunden. ${ }^{36}$ So waren die Landesherren von Tirol auch als Vögte in den geistlichen Territorien tätig ${ }^{37}$, und auch die Bistümer besaßen Gerichtsbezirke in der eigentlichen Grafschaft Tirol. ${ }^{38}$ Außerdem konnte bereits Meinhard II. im 13. Jahrhundert in Brixen und Trient weitgehende weltliche Herrschaftsrechte zu seinem Vorteil erhalten. ${ }^{39}$ In dieser Arbeit werden diese eigentlich reichsunmittelbaren Territorien miteinbezogen, da sie trotz ihrer de jure Selbstständigkeit mit den angrenzenden weltlichen Herrschaften verwoben waren und nicht ohne "das große Ganze“ betrachtet werden können. (Ein Beispiel: Das Hochstift Freising gehörte weder zu Bayern noch zu Tirol, das Stift besaß jedoch, wie auch die Bistümer Brixen und Trient, Besitzungen in Tirol, die in Hochgerichtsbarkeitsangelegenheiten einem Tiroler Landesgericht unterstanden und nur die Niedergerichtsbarkeit eigens ausüben durften. In Bayern besaß das Stift ebenso Hofmarken, die dem bayerischen Herzog unterstanden. ${ }^{40}$ )

34 Christian Wieland, Die Ausnahme in der Sprache des Allgemeinen. Bayerischer Adel und Gericht im 16. Jahrhundert, in: Adel und Adelskultur in Bayern, hrsg. v. Walter Demel/Ferdinand Kramer (Zeitschrift für bayerische Landesgeschichte Beiheft 32), München 2008, S. 107-135, hier S. 111.

35 Reinhard Heydenreuter, Strafrechtspflege in den bayerischen Besitzungen des Hochstifts Freising, in: Hochstift Freising. Beiträge zur Besitzgeschichte, hrsg. v. Hubert Glaser, München 1990, S. 217-228, S. 220.

36 Palme, Frühe Neuzeit, S. 92.

37 Ebd., S. 97.

38 Ebd., S. 99-101. Schennach, Gesetz und Herrschaft, S. 83.

39 Johanek, Adel in den österreichischen Ländern und in Tirol, S. 23 f.

40 Heydenreuter, Strafrechtspflege, S. 221, 228. 
Die Tiroler Landesordnung von 1526, entworfen von Michael Gaismair und entstanden während der Bauernkriege, war „der Versuch einer Neuordnung der Gesellschaft auf Dauer ${ }^{41}$. Im vierten Artikel heißt es:

„[Es] sollen alle [besonderen] Begünstigungen abgeschafft werden, denn sie sind wider das Wort Gottes und verfälschen das Recht, das besagt, dass niemand vor anderen bevorzugt werden soll. ${ }^{\text {"2 }}$

Zwar war diese Landesordnung eine Utopie ${ }^{43}$ und wurde bereits 1532 durch eine neue Landesordnung ersetzt, als Ferdinand I. wieder in einer stärkeren Position war. Dennoch war der Tiroler Adel von der Radikalität Gaismairs geschockt, sie hinterließ ein Trauma, in dessen Folge der Adel katholisch blieb, da das republikanische Modell Gaismairs stark von reformatorischen Ideen und dem Vorbild Zwingli und Zürich geprägt war. Dadurch entging der Tiroler Adel auch der späteren Säuberung durch die Habsburger mit dem Ziel, den protestantischen Adel zurückzudrängen, nachdem dieser offen gegen sie opponiert hatte. ${ }^{44}$

In den österreichischen Erbländern und damit auch in Oberösterreich bzw. Tirol und den Vorlanden ist eine hohe Geschlossenheit der Territorien zu bemerken, die auch dem Landadel zu verdanken sei, der das Land durch Bewusstsein und Anspruch verkörperte. ${ }^{45}$ Insgesamt ist zum Tiroler Adel zu sagen, dass er seit dem Mittelalter hauptsächlich aus der Ministerialität hervorging und in der Folge aufgrund der starken Stellung des Landesherren politisch geschwächt war. Diese überlegene Position des Grafen von Tirol fußte auch in der guten Finanzlage, da der Landesherr durch den regen Warenverkehr durch Tirol hohe Zolleinnahmen aufweisen konnte und dementsprechend wenig auf Zuwendungen des Adels hoffen musste. ${ }^{46}$

Ein weiterer Faktor, der die politische Macht des Adels, der auch im Vergleich zu Bayern in Tirol eine viel geringere Rolle spielte, sehr stark einschränkte, waren die Landstände:

„In den Tiroler Landständen waren nicht nur - wie in Steiermark, Kärnten und Krain auch - Hoch- und Niederadel in einer Bank vereint, sondern neben ihnen standen noch die landesherrlichen bäuerlichen Immediatuntertanen, Täler und Gerichte und damit waren auch Einschränkungen adliger Gerichtsbarkeit verbunden." ${ }^{47}$

Ein Akteur, der dem niederen Adel entgegenkam, war der Landeshauptmann. Dieser, vom Landesfürsten bestellte und mit Hofrecht ausgestattete "landeshauptmann an der Etsch und burggraf von Tirol“ war zunächst ein Vertreter bzw. Statthalter des Landesfürsten, wenn dieser nicht anwesend war. Um 1500 wandelte sich dessen „politische Ausrichtung

41 Michael Gaismair, Landesordnung für Tirol (1526). Einleitung von Nils Petersen, in: Die Entstehung einer sozialen Ordnung Europas. Historische Studien und exemplarische Beiträge zur Sozialreform im 16. Jahrhundert, hrsg. v. Theodor Strohm/Michael Klein Neröffentlichungen des Diakoniewissenschaftlichen Instituts an der Universität Heidelberg 22), Heidelberg 2004, S. 266-276, hier S. 267.

42 Gaismair, Landesordnung für Tirol, S. 272.

43 Johanek, Adel in den österreichischen Ländern und in Tirol, S. 25.

44 Ebd.

45 Ebd., S. 17

46 Ebd., S. 23

47 Ebd., S. 24. 
[...] vom Interessenvertreter der Grafen von Tirol hin zu einem Amt, das sich stärker den Interessen der Stände verpflichtet sah." ${ }^{48}$ Der Landeshauptmann sei demnach als vermittelnde Person zwischen Landesherr und Landständen anzusehen. ${ }^{49}$

\section{Steuerprivilegien}

Ursprünglich war der Gedanke der Steuer ein freiwilliger, erst spät hat er sich zum Zwang entwickelt. Diesen Freiwilligkeitsgedanken kann man auch an den Namen der frühesten Steuern ablesen: „precaria”, „petitio“ oder „bede“ lassen sich mit „Bitte“ oder "Geschenk" übersetzen. Das lässt darauf schließen, so Barbara Painer in ihrer Dissertation zum Steuerwesen in Meran, dass Steuern anfänglich zum einen eben freiwillig waren, zum anderen aber an einen bestimmten Anlass gebunden und damit nicht regelmäßig zu leisten waren. ${ }^{50}$

Eine wichtige Entwicklung hin zur allgemeinen Steuer stellt der Gemeine Pfennig dar, der 1495 auf dem Wormser Reichstag beschlossen wurde, zusammen mit einer Reihe weiterer Reformen auf Reichsebene, wie das Reichskammergericht und den Ewigen Landfrieden, mit dem auch das Verbot der Fehde einherging, woran auch die zunehmende Institutionalisierung und Staatsbildung abgelesen werden kann. Die Schaffung des Reichskammergerichtes als Institution und das Verbot der adeligen Selbstjustiz, der Fehde, hängen dabei ursächlich und kausal zusammen. ${ }^{51}$

Steuern waren in der Frühen Neuzeit schwierig durchzusetzen und mussten daher Voraussetzungen zur Rechtfertigung erfüllen: Sie mussten dem Gemeinwohl dienen und zweckgebunden sein. Daher mussten Steuern auch "gerecht" sein, und das bedeutete auch, dass Befreiungen von Steuern gewährt wurden. Das betraf zunächst die Geistlichkeit, aber auch Kranke, Blinde, Witwen, Waisen bzw. als bedürftig angesehene Personen. ${ }^{52}$

In der Frühen Neuzeit waren Steuern die Ausnahme, nicht die Regel. Sie mussten, wie bereits erwähnt, zweckgebunden sein: Um Straßen zu erhalten und auszubauen, wurde beispielsweise Maut oder Zoll verlangt. Gemäß dieser Vorstellung musste, damit die Steuer gerechtfertigt war, etwas im Gegenzug erhalten werden. Dieses Prinzip ist dem der Grundherrschaft ähnlich: Ein Bauer begab sich unter den Schutz eines Adeligen und durfte daher von diesem erwarten, dass er für die Sicherheit des Untertanen sorgte. Der Bauer hatte daher keinen Militärdienst zu leisten, denn der Grundherr war für die Aufstellung einer eigenen „Armee“ zuständig. Für diesen Schutz musste der Bauer Abgaben bzw. Fronen leisten. Das waren beispielsweise Dienstleistungen für den Grundherrn, wie etwa die Bestellung seiner Felder, Aushilfe bei Jagdgesellschaften des Adeligen oder die

48 Astrid von Schlachta, Das Amt des Landeshauptmanns - Verwaltung und Politik in Tirol im 18. Jahrhundert am Beispiel Paris Dominkus von Wolkenstein-Trostburgs und Paris von Wolkenstein-Rodeneggs, in: Die Wolkensteiner. Facetten des Tiroler Adels in Spätmittelalter und Neuzeit, hrsg. v. Kurt Andermann/Gustav Pfeifer (Veröffentlichungen des Südtiroler Landesarchivs 30), Innsbruck 2009, S. 345-359, hier S. 349.

49 Ebd.

50 Painer, Geschichte des Steuerwesens der Stadt Meran, S. 2

51 Wieland, Die Ausnahme in der Sprache des Allgemeinen, S. 121

52 Körner, Steuern und Abgaben in Theorie und Praxis, S. 57 f. 
Aufzucht der Jagdhunde. Unter Abgaben ist beispielsweise der Zehnt zu verstehen, der zumeist in Naturalien geleistet wurde.

Gemäß der Grundherrschaft leitete sich der Adel auch sein Privileg oder Vorrecht ab, selbst keine Steuern an den Landesherrn zahlen zu müssen. Der Adel sah es nicht als standesgemäß an, Steuern zu bezahlen. Für eine regelmäßig zu leistende monetäre Steuer sah sich der landsässige Adel bereits genug belastet, da er für das an inn vergebene Lehen des Landesherrn in Form von Grund und Boden dem Landesherrn ja Militärdienst lieferte und daher für eine Steuer keine Gegenleistung vom Landesherrn bekommen hätte. Die Leistungen, zu denen der Adel bereit war, wurden auch "Rat und Hilfe" genannt. Sie in Form von Geld zu leisten, empfand der Adel aber als unter seiner Würde. ${ }^{53}$

Von diesem Gedanken waren auch die ersten Theorien der Forschung zur Entstehung von Steuern ausgegangen: Die Steuer sei in dem Fall eingehoben worden, wenn ein dem Kriegsdienst verpflichteter Untertan des Landesherrn diesen nicht leisten konnte oder wollte. Ein anderer Ansatz war, dass die Steuern zur Finanzierung der Gerichtsbarkeit durch den Adel entstanden. ${ }^{54}$

Dem entsprechend nahm der Adel die Steuerfreiheit als sein gutes Recht wahr. Die Privilegien, die der Adel - und auch der Klerus - in diesem Bereich genoss, waren nur schwer abzubauen, und diese Bemühungen dauerten bis ins 19. Jahrhundert. ${ }^{55}$ Diese Freiheit des Adels wurde in der Geschichtswissenschaft auch schädlich aufgefasst, so zum Beispiel bei Viktor Ernst: „Ihren negativen Ausdruck findet die bevorrechtete Stellung des Burgherrn in der Freiheit von allerlei Lasten und Beschwerden, die auf der Gemeinde ruhen: Das Schloß mit seinen Gütern ist regelmäßig frei von der jährlichen Steuer. “56

\section{Das Steuerprivileg in Bayern}

Noch gegen Ende des 15. Jahrhunderts lehnten sich einige Adelige gegen die Steuerpolitik des bayerischen Landesherrn auf und schlossen sich im Löwlerbund zusammen. Die Adeligen empfanden die Besteuerung der eigenen Hintersassen durch den Landesherrn als Zumutung, die „einer Leibeigenschaft des Adels gleich“ käme. In weiterer Folge ging die Auseinandersetzung mit dem Landesherrn jedoch für die Adeligen schlecht zu Ende, da der Herzog den Bund zerschlagen konnte. Der Grund dafür dürfte im unterschiedlichen Verhalten des Niederadels zu finden sein. So stand nicht der gesamte Adel hinter dem Bund, es war also ein Aufstand einiger weniger mit militärisch sehr beschränkten Mitteln. ${ }^{57}$

An diesem Konflikt zeigte sich bereits die beginnende Schwächung des bayerischen Adels, aber noch musste der Herzog auf die eigenständige Einhebung der Steuern verzichten: Der Adel hatte sich in Bayern Ende des 15. Jahrhunderts, spätestens im Laufe des 16. Jahrhunderts, nicht nur das Privileg, keine Steuern zahlen zu müssen, sondern auch im Zuge der Mitgliedschaft in den Landständen das Privileg zur Steuerbewilligung

53 Körner, Steuern und Abgaben in Theorie und Praxis, S. 59

54 Painer, Geschichte des Steuerwesens der Stadt Meran, S. 3 f.

55 Ebd., S. 6, 11

56 Ernst, Entstehung des niederen Adels, S. 26.

57 Schneider, Spätmittelalterlicher deutscher Niederadel, S. 520-522. 
und Steuerverwaltung erkämpft. Damit wurde der Landesfürst teils von den Landständen abhängig, doch hatten die Landstände und besonders der Adel ein Interesse daran, Steuern zu bewilligen, zumal sie die Hauptgläubiger des Landesfürsten waren und damit an kontinuierlichen Einnahmen desselben interessiert waren und es auch als nötig empfinden mussten, Steuern zu bewilligen, insbesondere wenn sich Kriege abzeichneten wie etwa zu Beginn des 17. Jahrhunderts am Vorabend des Dreißigjährigen Krieges. Dennoch waren Steuern für die Landstände der wichtigste Bereich, in dem sie mitbestimmen konnten bzw. durften und in dem sie den größten Einfluss gegenüber dem Landesherren hatten, zumal dieser Teil der Verwaltung im 16. Jahrhundert aus dem fürstlichen Verwaltungsapparat ausgegliedert wurde und damit zumindest die Zustimmung der Stände notwendig war. ${ }^{58}$

Der Adel musste aber in Bayern trotz seiner Freiheit gewisse Steuern bezahlen. An dieser Stelle sind vor allem die sogenannten Standsteuern zu nennen, die nicht auf die Untertanen umgelegt werden durften. Zwar war hier der Adel nur mit zehn Prozent belastet, während Städte und Märkte vierzig Prozent und Geistlichkeit fünfzig Prozent der Standsteuer aufbringen mussten, doch beliefen sie sich allein während der Regierungszeit von Albrecht V. (1550 - 1579 Herzog in Bayern) auf 800.000 fl. ${ }^{59}$

Doch wie Richard Heydenreuter hervorhebt, stand der Anteil, den der Adel an den Standsteuern leistete, in keinem Verhältnis zu seinem Einfluss in der Landschaft, wo er die Hälfte aller Sitze innehatte. ${ }^{60} \mathrm{Im}$ beginnenden 17 . Jahrhundert zeigt sich zudem eine zunehmende Loslösung des Landesherrn von der Zustimmung durch die Landstände. "Der Landesherr", so Heydenreuter,

„beanspruchte die Steuer nun als Selbstverständlichkeit, da diese fast nur noch zur Rückzahlung und Verzinsung der Schulden und nur noch beschränkt zur Deckung künftiger Ausgaben diente. Die Landesherren beriefen sich bei ihren Forderungen auf Staatsnotwendigkeit bzw. auf Notstandsituationen [...], wobei seit dem 16. Jahrhundert viele Ausnahmesituationen propagandistisch überhöht wurden, um die Steuerbereitschaft zu fördern. "'b1

Eine weitere Steuer, die auch den bayerischen Adel traf, war die Rittersteuer, die aber an den Besitz einer Hofmark gebunden war. Diese durften die Adeligen aber, im Gegensatz zur Standsteuer, auf ihre Untertanen abwälzen ${ }^{62}$, und während des 15. Jahrhunderts konnten sie erreichen, dass ihr unmittelbares Vermögen von dieser Steuer befreit war. ${ }^{63}$ Margit Ksoll streift in ihrer Monographie zu den wirtschaftlichen Umständen des bayerischen Adels im 17. Jahrhundert die Thematik der Steuer nur am Rande und listet alle Ausgaben ihrer Untersuchungsfamilien auf64, wobei Steuern einen sehr geringen Anteil ausmachen. So kann davon ausgegangen werden, dass der bayerische Adel trotz

58 Kummer, Landstände und Landschaftsverordnung, S. 159-161.

59 Heydenreuter, Rechtstellung des landsässigen Adels, S. 56.

60 Ebd.

61 Ebd., S. $56 \mathrm{f}$

62 Ksoll, Die wirtschaftlichen Verhältnisse des bayerischen Adels, S. 20.

63 Kummer, Landstände und Landschaftsverordnung, S. 163.

64 Ksoll, Die wirtschaftlichen Verhältnisse des bayerischen Adels, S. 119-121, 135-137, 145-147, 149-150, 166-174, 191-194, 203-205. 
der zu leistenden Stand- und Rittersteuern nicht in großem Ausmaß von Steuern belastet war. Zum Beispiel erwirtschaftete die Familie der Grafen von Törring-Jettenbach und Törring zum Stain aus den Hofmarken Stain und Pertenstein im Jahr 1639 etwa 9000 fl. Die Rittersteuer machte für die Hofmarken Stain, Pertenstein und Pullach 82 fl. jährlich aus, weswegen auch noch um einen Nachlass von dieser Steuer gebeten wurde, wobei allerdings der Ausgang dieser Bitte nicht bekannt ist. ${ }^{65}$

Zur selben Zeit, als sich der Adel und auch die Geistlichkeit von den Landsteuern befreien und privilegieren lassen konnten, kam auch die Freiheit hinzu, in den Hofmarken die eigenen Untertanen besteuern und selbstständig einheben zu dürfen, jedoch mussten die Einnahmen an das Landsteueramt abgeführt werden. ${ }^{66}$

Zusätzlich zu den bereits erwähnten direkten Steuern kamen indirekte Steuern wie etwa Maut oder Zölle. Die Einhebung und Verwaltung dieser Abgaben lag nicht in der Hand des landsässigen Adels bzw. der Landstände, sondern wurde aufgrund von Privilegien des Landesherrn von diesem eingehoben. ${ }^{67}$ Der niedere Adel dürfte daher von diesen indirekten Steuern nicht befreit gewesen sein.

\section{Das Steuerprivileg in Tirol}

Unter der Herrschaft von Maximilian I. kam es bereits aufgrund seines Krieges gegen Venedig zu einer sehr hohen Steuerlast. Zu diesem Zweck wurde der Landtag häufig einberufen, um neue Steuern zu bewilligen, die Maximilians Krieg finanzieren sollten. ${ }^{68}$

Aufgrund der zu leistenden Steuern kam es auch zu Konflikten mit dem Adel. Ein Beispiel dafür bildet die Stadt Bruneck, wie Stephanie von Wenzl in ihrer Diplomarbeit herausgearbeitet hat: Zwar lebte der Adel vornehmlich auf dem Land, da Adeligen aus standesmoralischer Sicht der Handel bzw. eine Kaufmannskarriere verwehrt war und sie daher nicht in die Städte zogen. Dennoch versuchten sie, dies teilweise zu umgehen und besaßen häufig auch in den Städten Häuser. Die in der Stadt eingehobenen Steuern führten in der Folge zu einigen Konflikten um die Steuerfreiheit des Adels. Die Steuerfreiheit, so erscheint es, stand in den Städten stärker zur Disposition als auf dem Land. Ein Grund dafür könnte der Aufstieg des Bürgertums in den Städten sein, das zum Teil erheblichen Wohlstand erlangt hatte und dem die Privilegien des Adels ein Dorn im Auge waren. ${ }^{69}$ Auf der anderen Seite gab es eine nicht unwesentliche Zahl an nobilitierten bürgerlichen Familien in der Stadt, die daher auch mit Privilegien und Freiheiten ausgestattet waren. ${ }^{70}$

In der Folge wurden Adelige zur Leistung des Sitz- bzw. Hilfgeldes herangezogen, während sie von der Stadtsteuer - die nur dann verlangt wurde wenn einem Gewerbe nachgegangen wurde - sowie auch von der Haussteuer befreit waren. Das Sitz- oder Hilfsgeld war demnach ein Ersatz für die Nichtleistung der anderen städtischen Steuern,

65 Ksoll, Die wirtschaftlichen Verhältnisse des bayerischen Adels, S. 149.

66 Kummer, Landstände und Landschaftsverordnung, S. 163.

67 Ebd., S. 162.

68 Palme, Frühe Neuzeit, S. 13.

69 Wenzl, Stadt und Adel in Bruneck, S. 52.

70 Ebd., S. 32 
die Wenzl als "Nutzungsgebühr für die Einrichtungen der öffentlichen Infrastruktur" charakterisiert hat. ${ }^{71}$

Zu diesen Konflikten nach unten kam auch ein Konflikt mit dem Landesfürsten, der auch Klerus und Adel von ihren Privilegien zu lösen versuchte, während der Adel sich die Befreiungen bestätigen lassen wollte. ${ }^{72}$ Das hatte zur Folge, dass sich Adelige weigerten, Steuern zu bezahlen, obwohl die Steuern, um die es zumeist ging, einen sehr geringen Anteil aufwiesen. ${ }^{73}$ Es scheint beiden Konfliktparteien um das Prinzip gegangen zu sein. ${ }^{74}$

Auch in Meran kam es zu Konflikten des Adels mit dem Rat und Bürgerausschuss - sie legten die Steuern der Stadt fest - aufgrund der Genusssteuer, die auch der Adel zu bezahlen hatte und wo sich schließlich die Stadt durchsetzte: "Trotz aller Gegenwehr wurde der privilegierte Stand auch zur Zahlung dieser Steuer herangezogen, schließlich hatte er dieselben Vorteile in der Stadt zu wohnen, wie der Bürger und gemeine Inwohner."75

Der Adel kam in Tirol aufgrund der erhöhten finanziellen Bedürfnisse und einer Änderung der Steuerpolitik des Innsbrucker Hofes vermehrt unter steuerliche Belastungen, wobei der Adel, so Marcello Bonazza, unterschiedlich reagierte: Während sich der Adel, je näher er an Innsbruck lebte, tendenziell fügte, wurde vor allem im südlichen Teil des Landes die Zahlung von Steuern eher verweigert. Der Adel reagierte nicht als geschlossene homogene Gruppe, sondern wie Bonazza hervorhebt, durch individuelle Verhaltensweisen. ${ }^{76}$

Betrachtet man die Ergebnisse, die Barbara Painer zur Steuergeschichte Merans präsentiert, zeigt sich außerdem, dass Steuerfreiheit kein ausschließliches Privileg des Adels und des Klerus war. Vor allem in Städten gab es eine Reihe von Personen oder auch Berufsgruppen, die teilweise oder gänzlich von Steuern befreit sein konnten. Dabei ist auffällig, dass häufig Stadtbedienstete keine Steuern zu begleichen hatten. Zwar werden die betreffenden Personen als steuerpflichtig geführt, mussten jedoch nichts bezahlen. Wächter, Weinmesser, Lehrer, Metzger, Apotheker, Organisten, Bildhauer oder auch Ärzte konnten beispielsweise befreit sein. ${ }^{77}$

Im von Walter Schomburg herausgegebenen Lexikon zur Steuer- und Zollgeschichte wird eine so genannte Adelssteuer angeführt: „eine in Tirol erhobene Landsteuer von der Rente des Grundbesitzes"78. Doch leider bleibt das Nachschlagewerk an dieser Stelle sowohl eine Angabe zum Zeitpunkt dieser Steuer als auch zu weiterführender Literatur schuldig. Trotz Bemühungen konnten kaum weitere Hinweise für diese Steuer gefunden werden. Es könnte jedoch sein, dass Schomburg hier auf die Land- bzw. Gewaltsteuer

71 Wenzl, Stadt und Adel in Bruneck, S. $52 \mathrm{f}$.

72 Ebd., S. 55

73 Ebd., S. 58. Eine ausführliche und lesenswerte Beschreibung eines Beispieles von Auseinandersetzungen um das Bezahlen von Steuern in der Stadt Bruneck in der zweiten Hälfte des 16. Jahrhunderts kann bei Wenzl gefunden werden. Ebd., S. 58-90.

74 Zu diesem Schluss kommt auch Wenzl, die die Konflikte auch mit den ungenauen Definitionen sowohl der adeligen Freiheiten als auch den Aufgabenbereichen der Stadtverwaltung erklärt: Ebd., S. 91.

75 Painer, Geschichte des Steuerwesens der Stadt Meran, S. 53.

76 Bonazza, La nobiltà trentino-tirolese, S. 112.

77 Painer, Geschichte des Steuerwesens der Stadt Meran, S. 153, 199-231.

78 Walter Schomburg, Lexikon der deutschen Steuer- und Zollgeschichte. Abgaben, Dienste, Gebühren, Steuern und Zölle von den Anfängen bis 1806, München 1992, S. 8. 
hinweist, wie sie ähnlich auch in Bayern existierte, von der der dortige Adel jedoch befreit war. In Tirol gab es diesbezüglich Konflikte. So war der Adel, wie auch die Geistlichkeit, nicht damit einverstanden, dass ihre Besitzungen, gemessen am Ertrag, besteuert werden sollten. Eine ähnliche, vermögensbezogene Steuer wurde auch in der Stadt Bruneck, sowohl für Adel als auch Bürger, eingehoben. ${ }^{79}$ Ob Schomburg diese Steuer gemeint hat, ist fraglich. Die Ausführungen von Werner Köfler kommen der Sache dagegen näher, wonach diese Steuer eine Geldleistung anstatt zu stellender Soldaten sei, weswegen sie auch in Knechten gerechnet wurde. ${ }^{80}$

\section{Vergleich}

Im Steuerwesen hat sich gezeigt, dass der Adel zwar großteils von Steuern befreit war. Dies musste aber nicht heißen, dass er keine Steuern zu bezahlen hatte. Zwar waren sie meist gering, doch entzündeten sich daran häufig Konflikte. Gerade in der Stadt waren diese Auseinandersetzungen vorprogrammiert, typisch und häufig: „Während der Adel sich in seinen Privilegien nicht beschneiden lassen wollte, versuchte die Stadt, den Adel in die Steuerleistungen einzubinden; dabei ging es Bürgermeister und Rat vor allem auch um die Behauptung ihrer ohnehin bereits eingeschränkten Kompetenzen. “81

Zudem wurde herausgestellt, dass nicht nur Adelige allein befreit von Steuern sein konnten, sondern auch verschiedene, vor allem in Städten angesiedelte Berufsgruppen und bedürftige Menschen, denen eine Steuerleistung nicht zugemutet werden konnte. ${ }^{82}$ Im Bereich der Steuerbewilligung hatte der Adel jedoch stärker die Zügel in der Hand, wobei dies auch nur für Tirol ${ }^{83}$ und Bayern auffällig sein könnte; für Territorien in Schwaben stimmt das beispielsweise nicht:

„Eine empfindliche Lücke in den Rechten des Burgherrn bildet in der Regel das Fehlen des Steuerrechts, das im Hauptgebiet Schwabens höchstens ausnahmsweise in die Hand des niederen Adels gelangt ist. Insbesondere ist die älteste Steuer, die Bede meist nicht in die Hand des Burgherren kommen [sic], sondern wird von seinem Dorfe an den zuständigen Grafen gezahlt. "84

\section{Gerichtsbarkeit}

Erst die mit dem Grundbesitz verbundenen Herrschaftsrechte machten das wichtigste Charakteristikum des adeligen Besitzes aus, was sich konkret in der Besetzung von Gerichten

79 Wenzl, Stadt und Adel in Bruneck, S. 55 f.

80 Siehe dazu: Werner Köfler, Land - Landschaft - Landtag. Geschichte der Tiroler Landtage von Anfängen bis 1808 (Veröffentlichungen des Tiroler Landesarchivs 3), Innsbruck 1985, S. 136.

81 Wenzl, Stadt und Adel in Bruneck, S. 95.

82 Zur Befreiung verschiedener Gruppen siehe auch: Schomburg, Steuer- und Zollgeschichte, S. 362-363.

83 Für Tirol wurde das im Text aus Platzgründen zwar nicht explizit herausgearbeitet, doch funktionierte die Steuerbewilligung ähnlich wie in Bayern über die Landstände, siehe hierzu: Köfler, Land - Landschaft Landtag, S. 96-174.

84 Ernst, Entstehung des niederen Adels, S. 28. 
oder in der selbstständigen Ausübung des Richteramtes äußert. Auch die Einbehaltung der vom Gericht ausgesprochenen Strafen war mit diesem Recht eingeschlossen. ${ }^{85}$

In der Frühen Neuzeit wurden erste Institutionen geschaffen, und das Prinzip der Staatlichkeit begann sich zu entwickeln. Ein Anzeichen dafür ist die Schaffung des Reichskammergerichtes, beschlossen auf Initiative von Kaiser Maximilian I. auf dem Wormser Reichstag. In der Folge wurde dieses Datum, 1495, als sehr bedeutend wahrgenommen und häufig für die Abgrenzung von Mittelalter und Neuzeit verwendet. ${ }^{86}$ Das Reichskammergericht bleibt jedoch nur eine von vielen Möglichkeiten, eine Epochengrenze zu ziehen, und häufig ist es nur Zufall, dass gerade das Ereignis ausgewählt wird, das ungefähr in die Zeit fällt, in der eine Grenze gezogen werden soll. In diesem Fall wurden viele Ereignisse rund um das Jahr 1500 genannt, angefangen vom Druck des ersten Buches Mitte des 15. Jahrhunderts bis hin zum Augsburger Religionsfrieden 1555.

Tatsache ist aber, dass das Reichskammergericht Ausdruck einer Institutionalisierung war, mit deren Entstehung eine Professionalisierung im Bereich der Gerichtsbarkeit einherging. Das römische Recht wurde verstärkt herangezogen und rezipiert, was ausgebildete Juristen verlangte. Da der Adel sich dem häufig verwehrte oder diese Aufgabe als nicht standesgemäß ansah, waren das Anrecht auf die Rechtsprechung und die Ausübung der Gerichtsbarkeit immer schwieriger beizubehalten. Das hatte zur Folge, dass aus bürgerlichen, geschulten Juristen ein neuer Amtsadel entstand. ${ }^{87}$

Dennoch waren Grundherrschaften, die nach wie vor hauptsächlich in der Hand des alten landsässigen Adels waren, in der Regel mit Gerichtskompetenzen ausgestattet. Im Laufe des 17. Jahrhunderts wurden schließlich Grundherren häufig aufgefordert, ihre Tätigkeit als Richter nicht mehr selbst auszuüben. So geschehen beispielsweise im Elsass im Jahr $1686 .{ }^{88}$

Da der Adel sich vornehmlich über die Ausübung von Herrschaft definierte und sich diese in der Ausübung der Gerichtsbarkeit am eindrücklichsten und prestigeträchtigsten zeigte, war der Adel angehalten, um seines Standes willen, sich die Vorrechte zu erhalten und sie nicht in bürgerliche Hände abzugeben. Wie das in den Territorien Bayern und Tirol aussah, soll in den nachfolgenden Abschnitten erläutert werden.

Zuvor muss an dieser Stelle allerdings noch kurz auf den Unterschied der Hoch- und der Niedergerichtsbarkeit eingegangen werden: In der Regel hatte der landsässige Adel zumindest die Niedergerichtsbarkeit inne, während der Landesherr sich die Hochgerichtsbarkeit in der Frühen Neuzeit meist vorbehielt. Der Unterschied lag maßgeblich in der Schwere der begangenen Straftaten. Deswegen wird im Fall der

85 Ernst, Entstehung des niederen Adels, S. $10 \mathrm{f}$.

86 Wieland, Die Ausnahme in der Sprache des Allgemeinen, S. 120.

87 Barbara Kink, Adelige Lebenswelt in Bayern im 18. Jahrhundert. Die Tage- und Ausgabebücher des Freiherrn Sebastian von Pemler von Hurlach und Leutstetten (1718-1772) (Studien zur bayerischen Verfassungs- und Sozialgeschichte 26), München 2007, S. 52.

88 Erich Pelzer, Der elsässische Adel im Spätfeudalismus. Tradition und Wandel einer regionalen Elite zwischen dem Westfälischen Frieden und der Revolution (1648-1790) (Ancien Régime, Aufklärung und Revolution 21), München 1990, S. 193 f. 
Hochgerichtsbarkeit auch häufig von Blut- oder Malefizgerichtsbarkeit gesprochen. Welche Strafbestände das genau umfasste, konnte variieren, jedenfalls musste das Gericht, in dem die Straftat stattgefunden hatte bzw. das den Fall behandelte, zunächst prüfen, ob es sich um ein Malefizverbrechen handelte. War dies der Fall, und das Gericht war nicht mit den entsprechenden Befugnissen ausgestattet, musste der Fall an die zuständigen höheren Gerichte übergeben werden. ${ }^{89}$

\section{Die Gerichtsbarkeit in Bayern}

Um die Gerichtsbarkeit in Bayern erfassen zu können, muss zunächst das Prinzip der Hofmarken erklärt werden, die für Bayern eine zentrale Rolle einnahmen, das "typisch bayerische [...] Niedergericht" ${ }^{\prime 90}$. Klaus Kopfmann definiert die Hofmark wie folgt:

„Unter der Bezeichnung Hofmark versteht man teils geschlossene, teils offene Herrschaftsgebiete, die zumeist ein oder mehrere Dörfer umfassten und in denen in der Regel geistliche oder weltliche Hofmarkherren berechtigt waren, die Hofmarkprivilegien auszuüben. “91

Diese Privilegien umfassten die Niedergerichtsbarkeit sowie Prozesse um liegendes Gut, so genannte Gantprozesse, „die freiwillige Gerichtsbarkeit, die allgemeine Verwaltung und Polizeihoheit [...], ferner das Steuerveranlagungs- und Musterungsrecht, der Anspruch auf unentgeltliche Scharwerksdienste sowie die Ausübung der Niederjagd." ${ }^{\text {"2 }}$

Welch zentrale Rolle die Hofmark in Bayern spielte, zeigt auch die Aufstellung von Kopfmann, wonach Ende des 17. Jahrhunderts knapp fünfzig Prozent der ländlichen Bevölkerung Bayerns einem Hofmarkherren Untertan waren. Gezählt wurden für das Jahr 1598880 verschiedene Hofmarken. ${ }^{93}$

Die Hofmark war die Grundlage des Adels in Bayern, durch sie war der Adel berechtigt, in der Landschaft im Ritterstand zu sein. In der Landschaft hatte der Adel auch die Möglichkeit, politisch tätig zu sein und auch in innen- wie außenpolitischer Hinsicht gegenüber dem Landesherrn eine gewichtige Stellung einzunehmen, maßgeblich durch das Bewilligungsrecht der finanziellen Mittel des Herzoges. Dieser begann im 15. Jahrhundert mit dem Versuch, die Rechte der Landschaften zu beschränken, durch die Förderung von nichtbayerischem Adel. ${ }^{94}$

89 Schennach, Gesetz und Herrschaft, S. 77

90 Wolfgang Schuster, o.T., in: Adel in Bayern. Ritter, Grafen, Industriebarone, hrsg. v. Wolfgang Jahn/Margot Hamm/Evamaria Brockhoff, Stuttgart 2008, S. 111

91 Klaus Kopfmann, Wirtschaftliche Grundlagen - die Hofmarken, in: Adel in Bayern. Ritter, Grafen, Industriebarone, hrsg. v. Wolfgang Jahn/Margot Hamm/Evamaria Brockhoff, Stuttgart 2008, S. 104-105, hier S. 104.

92 Ebd. Zu den einzelnen Rechten, die mit der Hofmark verbunden waren, siehe auch Heydenreuter, Rechtstellung des landsässigen Adels, S. 57. Heydenreuter merkt hier zurecht an, dass auch andere, nicht adelige Hofmarkseigentümer, dieses Recht hatten, es sich hierbei also nicht um ein rein adeliges Privileg handelte, sondern dass es auch Bürgern offen stand. Dass es diesen auch möglich war, in den Besitz einer Hofmark zu gelangen, zeigt auch, wieso die Hofmark, wie unten erwähnt, eine Möglichkeit zum Aufstieg in den Adel war.

93 Kopfmann, Wirtschaftliche Grundlagen, S. 104.

94 Andrian-Werburg, Der altbaierische Adel, S. 51-53. 
Ab dem beginnenden 16. Jahrhundert verlor der "altbaierische“ Adel an Bedeutung, was sich im weitgehenden Verlust der hohen Gerichtsbarkeit äußert. Bisher hatte sich der Adel den herzoglichen Landgerichten verwehren können. Durch die zunehmende Ausdünnung des alten Adels kamen die Herrschaften und damit viele Hofmarken zum neuen Adel. Damit konnten sich die Wittelsbacher als Landesherren vorwiegend die Hochgerichtsbarkeit sichern, während die bisher mit der Hochgerichtsbarkeit ausgestatteten Herrschaften zu Hofmarken wurden und damit nur noch die Niedergerichtsbarkeit ausüben durften. ${ }^{95}$ Später waren vor allem der Dreißigjährige Krieg und die mit diesem einhergehende hohe Verschuldung des Adels ein weiterer Schub zur Verlagerung der Herrschaften in die Hände des neuen Adels. Dementsprechend war die Hofmark auch Trittbrett für die Nobilitierung einer Familie und den Aufstieg in den niederen Adel. ${ }^{96}$

Die Landschaften kamen aber 1508 zu ihrer größten Errungenschaft gegenüber dem Herzog, trotz dessen Versuchen, diese zu schwächen: die Erklärte Landesfreiheit der Ritterschaft. ${ }^{97}$ Die Erklärte Landesfreiheit definierte die Hofmarken und die Rechte, die mit inr einhergingen. Neben der niederen Gerichtsbarkeit fielen hier auch die Jagdrechte des Hofmarkinhabers hinein. Bis ins 19. Jahrhundert blieb die Erklärte Landesfreiheit die Grundlage des Gerichtsbarkeitsrechtes in Bayern und legte das Verhältnis fest, in dem landsässiger Adel und Landesherr zueinander standen. ${ }^{98}$ Von der Forschung wurde der Erklärten Landesfreiheit der Beiname „bayerische Bill of Rights“ aufgrund ihrer zentralen Bedeutung verliehen. ${ }^{99}$

Ein wichtiges Datum für den bayerischen Adel stellt auch das Jahr 1557 dar. In diesem Jahr wurde der 60. Freibrief ausgestellt, in dem die Erklärte Landesfreiheit bestätigt wurde und dem bayerischen Altadel die so genannte Edelmannsfreiheit ${ }^{100}$ gewährt wurde, durch die sich der Altadel gegenüber dem neuen Adel absetzen konnte und "zu einer in sich geschlossenen Gemeinschaft" wurde. ${ }^{101}$ Nun durften die Hofmarkherren, die die Edelmannsfreiheit besaßen auch über einschichtige Güter die Niedergerichtsbarkeit ausüben. ${ }^{102}$

95 Andrian-Werburg, Der altbaierische Adel, S. 49. Richard Heydenreuter hat diejenigen adeligen Besitztümer in Bayern für den Zeitraum 16.-18. Jahrhundert herausgearbeitet, die sich die hohe bzw. Blutgerichtsbarkeit erhalten konnten: Heydenreuter, Rechtstellung des landsässigen Adels, S. 64-75.

96 Dietmar Stutzer, Zwei Hofmarken im Vergleich: Stachesried und Hilling, in: Adel in Bayern. Ritter, Grafen, Industriebarone, hrsg. v. Wolfgang Jahn/Margot Hamm/Evamaria Brockhoff, Stuttgart 2008, S. 105-111, hier S. 106

97 Andrian-Werburg, Der altbaierische Adel, S. 55.

98 Bavarikon, Die erklärte Landesfreiheit des Herzogtums Bayern von 1508, [http://bavarikon.de/de/ bookviewer/kpbO-GDA-OBJ-0000000000000024_00001], 2013, eingesehen 19.2.2014.

99 Andrian-Werburg, Der altbaierische Adel, S. 55.

100 Mit der Edelmannsfreiheit ausgestattete Adelige waren von landesherrlichen Fronden befreit, hatten das Recht selbst solche zu fordern und durften selbst die Untertanen besteuern und mustern. Dazu kam noch die Gerichtsbarkeit und das Jagdrecht. Schomburg, Steuer- und Zollgeschichte, S. 78. Ksoll, Die wirtschaftlichen Verhältnisse des bayerischen Adels, S. 12.

101 Andrian-Werburg, Der altbaierischeAdel, S. 55. Bavarikon, Der 60. Freiheitsbrief der bayerischen Landstände: Verleihung der Edelmannsfreiheit, [http://bavarikon.de/de/image/kpbO-GDA-OBJ-0000000000000026], 2013, eingesehen 19.2.2014.

102 Kopfmann, Wirtschaftliche Grundlagen, S. 104. Unter einem einschichtigen Gut verstand man in Bayern ein Bauerngut, das zuvor nicht zum Einzugsbereich der Hofmark gehörte und direkt dem Landgericht unterstellt war. 
Andrian-Werburg bezeichnet die politische Macht des landsässigen Adels Mitte des 16. Jahrhunderts allerdings als

"ein deutliches Zurücktreten des altbaierischen Adels von der Politik des Herzogtumes [...], da das Herrscherhaus jetzt in steigendem Maß ausländischen, nichtdeutschen Adel ins Land zieht [...]. Dies und die bewusste Schaffung eines neuen Beamtenadels hat in kürzester Zeit bewirkt, daß der Begriff des bayerischen Adels, je nachdem ob er vor oder nach der Mitte des 16. Jahrhunderts angewendet wird, ganz verschiedene Vorstellungen erfordert. ${ }^{\prime 103}$

\section{Die Gerichtsbarkeit in Tirol}

In Tirol war die Gerichtsbarkeit ausgesprochen wenig in adeliger oder auch geistlicher Hand. Selbst die Niedergerichtsbarkeit war häufig den Gerichten vorbehalten, die verschiedene Titel und damit verschiedene Rechte haben konnten. Es existierten verschiedenste Formen nebeneinander: Landgericht, Stadtgericht, Marktgericht, Hofmark, Burgfrieden und Gericht. Nur das Landgericht hatte die Hochgerichtsbarkeit inne, alle anderen mussten nach kurzer Überprüfung, ob es sich beim jeweiligen Verfahren um eine Malefizangelegenheit handelte, den Fall an das Landgericht übergeben. Alle anderen Gerichte erhielten nur die niedere Gerichtsbarkeit zugesprochen. Der maßgebliche Unterschied dieser verschiedenen Formen ist vor allem in der flächenmäßigen Ausbreitung zu finden, wobei ein Burgfrieden häufig nur einige wenige Höfe umfasste. ${ }^{104}$

Über den Landgerichten stand noch die „oberste Appellationsinstanz": die Innsbrucker Regierung, die auch der Gerichtsstand für den Adel nördlich des Brenners war. Südlich des Brenners war dies das Hofrecht in Bozen bzw. Meran. ${ }^{105}$ Die Gerichtsbarkeitsrechte des Adels in Tirol waren bereits am Ende des 15. Jahrhunderts so gut wie nicht mehr existent. Schennach beschreibt die adelige Gerichtsbarkeit

„in vernachlässigbarem Umfang in manchen Burgfrieden. Der adelige Inhaber einer Burg konnte sich unter Umständen die Niedergerichtsbarkeit über die in der engsten Umgebung der Burg liegenden und dieser mit Leistungspflichten verbundenen Hofstellen erhalten (wobei keineswegs jeder Burgfrieden ein eigener, sich vom umgebenden Gericht abhebender Niedergerichtsbezirk sein muss)."106

In der Folge fasst er seine Erläuterungen zusammen:

„Insgesamt ist somit festzuhalten, dass nur sehr wenige geistliche und weltliche Herren eigene, von der Fläche und Einwohnerzahl her marginale Gerichtsbezirke ausbilden bzw. behaupten konnten. Die meisten Gerichte waren landesfürstlich - was keineswegs heißt, dass der Landesfürst sie in Eigenregie durch von inm eingesetzte Amtsträger verwalten lassen musste. “107

103 Andrian-Werburg, Der altbaierische Adel, S. 56.

104 Schennach, Gesetz und Herrschaft, S. 77.

105 Palme, Frühe Neuzeit, S. 96.

106 Schennach, Gesetz und Herrschaft, S. 79

107 Ebd. 
Dementsprechend wurden vom Landesfürsten, besonders noch unter Meinhard II. (1257-1295), Amtsträger (Richter) eingesetzt, die aus den Einnahmen, die das Gericht durch die laufenden Geschäfte erhielt, ein festes Gehalt bezog. ${ }^{108}$ Schulden und ständig benötigte Geldmittel zwangen den Landesfürsten aber schon bald dazu, Gerichtsrechte zu verpfänden, und zwar an Adelige, die Gläubiger des Landesherren:

„Zugrunde lag einer solchen Verpfändung stets ein Darlehen bzw. eine Schuldforderung des Adeligen an den Landesfürsten, wofür dem Gläubiger im Gegenzug die Nutzungen aus einer Gerichtsherrschaft überlassen wurden, bis das Schuldkapital samt Zinsen getilgt war. Eine solche Gerichtsherrschaft war dabei finanziell attraktiv - tendenziell ein Verlustgeschäft war allein die Ausübung der (prestigeträchtigen) Hochgerichtsbarkeit, während die Niedergerichtsbarkeit aufgrund der anfallenden Gerichtsgebühren und Bußgelder durchaus lukrativ war."109

Adelige hatten in Tirol weiters die Möglichkeit zum Zugriff auf die Gerichtsbarkeit durch das „Pfandlehen“. Dieses funktionierte wie folgt: Der Adelige gewährte dem Landesherren gegen die Belehnung mit einer erblichen Gerichtsherrschaft einen Kredit. Im Gegensatz zur Verpfändung, die jederzeit wieder entzogen werden konnte, war dieses System äußerst vorteilhaft und sehr sicher für den Adel. Da in der Folge einige Adelsfamilien sehr lange Zeit im Besitz einer Gerichtsherrschaft waren, entwickelten sich sogenannte "Dynastialgerichte“. Ein derartiges Lehen war für die adelige Seite „eine sichere Investition, die zudem prestigeträchtig war." ${ }^{110}$

Beispiele für derartige Dynasten sind die Familien von Wolkenstein, von Zenobio, von Künigl, von Hendl, von Khuen, von Lamberg oder von Trapp. Diese Familien hatten die Gerichtsrechte teils vom beginnenden 16. bis hinein ins 19. Jahrhundert inne und verfügten über beträchtliche Gebiete. ${ }^{111}$

Im Zusammenhang mit der Verpfändung von Herrschaften kam es auch zu Konflikten zwischen Landesherr, Geistlichkeit und Adel. An den Bischof von Brixen waren seit Anfang des 16. Jahrhunderts die vier Herrschaften Michelsburg, Uttenheim, Schöneck und Heinfels verpfändet. ${ }^{12}$ Nachdem Erzherzog Ferdinand 1567 Graf von Tirol geworden war, versuchte er diese wieder unter weltliche, landesherrliche Kontrolle zu bekommen und beauftragte reiche Tiroler Adelige, die betreffenden Gebiete abzukaufen, sehr zum Missfallen der Fürstbischöfe. Die Gerichtsbezirke konnten allerdings nur für zehn Jahre gehalten werden und wurden 1581 erneut für 25 Jahre an das Bistum Brixen verpfändet. ${ }^{113}$

108 Schennach, Gesetz und Herrschaft, S. 79 f.

109 Ebd., S. 80.

110 Ebd., S. $80 \mathrm{f}$.

111 Otto Stolz, Politisch-historische Landesbeschreibung von Südtirol. Zweiter Teil der Landesbeschreibung von Tirol (Schlern-Schriften 40), Innsbruck 1937, S. 37 f.

112 Palme, Frühe Neuzeit, S. 99.

113 Ebd., S. 101. 
Die Einschränkungen des Tiroler Adels in der Gerichtsbarkeit waren äußerst ungewöhnlich, wie Peter Johanek hervorhebt. Das lag vor allem daran, dass in den Landständen, wie bereits erwähnt, neben dem Adel auch Bauern, Märkte, Städte und Gerichte vertreten waren. ${ }^{114}$

\section{Vergleich}

In Bayern wurde interessanterweise durch die Edelmannsfreiheit von 1557 zwischen privilegiertem Adel und nicht privilegiertem Adel unterschieden. Erst nach Erlangung der Edelmannsfreiheit, durften viele Vorrechte genutzt werden, wodurch ein "einfacher ,edelmannsfreier' Adeliger besser gestellt sein konnte als ein Graf oder Freiherr, der nicht zum bayerischen Adel gehörte. "115 Eine solche Unterscheidung findet sich in Tirol nicht, jedoch unterstreichen die Erkenntnisse aus Bayern und Tirol, dass der Adel in sich nicht homogen war und auch in nahe beieinander liegenden Territorien teils große Unterschiede in den Rechten des Adels bestanden. Während in Bayern die Niedergerichtsbarkeit häufig in der Hand des landsässigen Adels war, war die Situation in Tirol wesentlich komplizierter. Flächenmäßig bedeutsame Gerichtsbarkeit trat in Tirol meist nur bei den "Dynastialgerichten" auf, die dann auch die hohe Gerichtsbarkeit haben konnten, sofern es sich um ein Landgericht handelte. In Bayern war die hohe Gerichtsbarkeit für den Adel ein fernes Ziel.

\section{Jagdrecht}

Die Jagd ist ausgesprochen eng mit Gerichtsbarkeit verbunden, da diese beiden Vorrechte des Adels meist miteinander einhergehen. Die Jagd selbst nimmt in der Kultur des Adels eine sehr wichtige Stellung ein. Sie ist "die adelige Betätigung par excellence“116:

Das Jagdrecht war nämlich ein Hoheitsrecht. Während dem Landesherrn die ,hohe Jagd' auf Rot-, Dam- und Schwarzwild sowie auch auf den Auerhahn vorbehalten war, durfte der landsässige Adel - soweit inm nicht auch die hohe Jagd zustand - lediglich die ,niedere Jagd' auf Hasen, Füchse, Dachse, Rebhühner und Enten ausüben. Die Zuordnung mancher Wildarten war unterschiedlich geregelt. Nach dem Jagdrecht durfte der Adel auf dem bäuerlichen Land jagen. ${ }^{117}$

Dieses Vorrecht für den Adel stand praktisch außer Zweifel, wie auch an den Forderungen der Bauern im Bauernkrieg gesehen werden kann oder auch an zahlreichen Publikationen, wie etwa den „XV. Bücher von dem Feldbaw”:

„Vnd endlich auch darumb / das in beschreibung dieser volkommenen Meyerey auch der Fürstlichen vn(n) Adelichen Ubung des Jagens / Hetzens / Beyssens /

114 Johanek, Adel in den österreichischen Ländern und in Tirol, S. 24

115 Heydenreuter, Rechtstellung des landsässigen Adels, S. 104 f.

116 Bieberstein, Adelsherrschaft und Adelskultur, S. 269.

117 Ebd., S. 280. 
vnd sonst Weydwercks gedacht / vnd deshalb billich eim Fürsten angetragen wirdt." 118

Dieses Zitat aus dem Vorwort zeigt eindrücklich, dass die Jagd im Denken und in Darstellungen der Zeitgenossen als etwas ausschließlich Adeliges verstanden bzw. propagiert wurde.

Wie an den Forderungen aus dem Bauernkrieg zu sehen ist, war die adelige Jagd zwar mit hohen Lasten für die Untertanen und insbesondere die Bauern verbunden, doch blieb das Vorrecht des Adels auf die Jagd zumeist unangetastet, wurde von bäuerlicher Seite respektiert und kaum in Frage gestellt. Man beschwerte sich über die Schäden, die einerseits die Jagdgesellschaften selbst an den Feldern der Bauern verursachten, andererseits über die Schäden, die das Wild anrichtete, da es den Untertanen häufig verboten war, das Wild auch nur zu vertreiben oder durch Hunde ihre Ernte zu schützen. ${ }^{119}$

Die Jagd konnte aber auch, wie Viktor Ernst herausstellt, dem Ortsadel oder der Gemeinde entzogen sein, auch wenn der Adel "die Jagd als Zubehör seiner Güter in Anspruch" nahm, wobei es allerdings in dem von Ernst untersuchten Raum Schwaben verhältnismäßig viele Gebiete gab, die noch die so genannte „Freie Pürsch“ besaßen. ${ }^{120}$

Unter der Freien Pürsch versteht man im Allgemeinen zwar nicht die Unbeschränktheit der Jagd, aber die Erlaubnis für zumindest die Mehrheit der Bevölkerung, jagen zu dürfen, was vor allem in Südwestdeutschland der Fall war, um Ulm und in Teilen des Schwarzwaldes. Die Freie Pürsch stand im Gegensatz zu der sonstigen Vormachtstellung des Adels und vor allem des hohen Adels im Jagdrecht, doch könnte die Entstehung der Freien Pürschen mehrere Gründe haben: Einerseits sah die Realität wohl kaum anders aus als in stark beschränkten Gebieten - die Herrschenden jagten am ausführlichsten. Andererseits scheint die Freie Pürsch vom Herzog von Württemberg beispielsweise "als Waffe gegen den ritterschaftlichen Adel und als Vehikel seiner vordringenden Landeshoheit" eingesetzt worden zu sein. Eine weitere Erklärung für die Freie Pürsch könnte die starke Zersplitterung der einzelnen kleinen Territorien in diesem Raum sein. ${ }^{121}$

\section{Das Jagdrecht in Bayern}

In der Regel besaß der bayerische niedere Adel, der im Besitz einer Hofmark war, auch das Recht, die niedere Jagd auszuüben. Hier zeigt sich auch die enge Verzahnung von Gerichtsbarkeit und Jagdausübung, die sehr häufig Hand in Hand gingen. Zum niederen Wild zählten (im Fall von Bayern): Vögel, Rehe, Füchse, Hasen und Wildschweine ${ }^{122}$, oder, umgekehrt ausgedrückt, alles Wild und Vögel, mit Ausnahme von Hirschen, Gämsen,

118 Charles Estienne/Jean Liébault, XV. Bücher von dem Feldbaw vnd recht volkommener Wolbestellung eines bekömmlichen Landsitzes / vnnd geschicklich angeordneten Meyerhofs oder Landguts / Sampt allem / was demselben Nutzes vnd Lusts halben anhängig, Straßburg 1598, S. VII.

119 Rösener, Die Geschichte der Jagd, S. 255.

120 Ernst, Entstehung des niederen Adels, S. 24.

121 Eckardt, Herrschaftliche Jagd, bäuerliche Not und bürgerliche Kritik, S. 42 f. Rösener, Geschichte der Jagd, S. $259 \mathrm{f}$.

122 Heydenreuter, Rechtstellung des landsässigen Adels, S. 80. 
Wildschweinen, Steinböcken, Reihern und Falken. ${ }^{123}$ Die teilweise Widersprüchlichkeit dieser beiden aus der Forschungsliteratur entnommenen Auflistungen zeigt, dass in Bayern nicht von einer einheitlichen Zusammenstellung der freigegebenen Jagdtiere gesprochen werden kann, da dies oft auch von Herrschaft zu Herrschaft, von Besitz zu Besitz, von Zeitpunkt zu Zeitpunkt divergieren konnte.

Interessant an der niederen Jagd in Bayern ist auch die strenge Unterscheidung innerhalb des Adels, wer wo jagen durfte. Wie bereits im Abschnitt zur Gerichtsbarkeit gezeigt, waren Inhaber der Edelmannsfreiheit mit den meisten Rechten ausgestattet. Sie durften auch außerhalb der Hofmarkgrenzen jagen, während Adelige ohne Edelmannsfreiheit nur innerhalb der eigenen Hofmark zur Jagd berechtigt waren und bürgerliche Hofmarkbesitzer gar nicht, nicht einmal auf ihrem Hofmarkbesitz. ${ }^{124}$

Diese Abstufungen und die damit einhergehenden Unterschiede im Umfang der zur Verfügung stehenden Jagdgebiete war Grundlage für unzählige Auseinandersetzungen des Adels, aber auch von dessen Untertanen mit der Verwaltung des Herzogs. ${ }^{125}$

Für das Herzogtum Bayern hat Monika Ruth Franz die beinahe identischen Landesordnungen von 1516 und 1520 ediert, in denen sich auch Abschnitte zum Jagdrecht befinden. Darin wird deutlich, wie sehr der Landesherr sein Recht, selbst kleine Tiere wie Hasen alleine zu jagen bzw. für alle, egal ob adelig oder nicht, ein Jagdverbot durchsetzen wollte:

„So ist hierauf mit Rate vnnserer Lanndtschafft vnser ernnstlich beuelh, will vnd maynung, das füran nyemandts, Er sey edl oder vnedl, hochs oder nyders stannds, in vnserm fürstenthomb die Hasen mit abschreckhen laussen, wonsässen, mit hürdt, täugken oder in schnu(e)ern, noch in kegeln vahen sol, sonder hyemit allermenigklich genntzlich vnnd gar verpotten sein vnnd pleiben." ${ }^{26}$

Doch wird die gesamte Landesordnung für Adelige (unter anderem) relativiert und abgeschwächt:

„Doch so(e)llen vorgeschribne Landpot, Satzung vnd ordnung alle samentlich vnd sonderlich [...], geistlichen und welltlichen, Edln vnd vnedln, Stetten vnnd Märckthen an jren freyhaiten, allten herbrachten gebreüchen, briefen, declarationen, Rechten vnnd Gerechtigkaiten [...] vnuergriffen, vnuerpfendt, vnenntgolten vnd on allen schaden sein.“"127

War also bereits zuvor die Freiheit, um in diesem Fall Hasen zu jagen, in eigenem Besitz, so durfte dies auch nach den Landesordnungen von 1516 und 1520 fortgesetzt werden. Auch Franz bekräftigt dies in ihrem Kommentar zur Landesordnung, merkt aber an, dass das Verbot der Hasenjagd für Adelige nur schwer mit dem 1516 zugesicherten Recht auf

123 Ksoll, Die wirtschaftlichen Verhältnisse des bayerischen Adels, S. 19

124 Heydenreuter, Rechtstellung des landsässigen Adels, S. 81. Ksoll, Die wirtschaftlichen Verhältnisse des bayerischen Adels, S. 19.

125 Heydenreuter, Rechtstellung des landsässigen Adels, S. 81.

126 Franz, Die Landesordnung von 1516/1520, S. 69.

127 Ebd., S. 103. 
die niedere Jagd, also auch auf Hasen, vereinbar war und dieser Abschnitt wahrscheinlich nur als Appell an den Adel gedacht war. ${ }^{128}$

Nicht gültig war dies jedoch in Bezug auf den Landfrieden, der von Kaiser Maximilian am Wormser Reichstag 1495 initiiert wurde. Hier wurden alle Privilegien, Freiheiten oder alten Übereinkommen ausdrücklich außer Kraft gesetzt. ${ }^{129}$

Generell, so Wilhelm Eckardt in seiner für die Jagdgeschichte bedeutsamen Monographie, hatte der Landesherr in Bayern Mühe, sich die alleinigen Jagdrechte zu sichern. Der landsässige Adel habe seine Privilegien hier „eifersüchtig bewacht“. ${ }^{130}$ Deshalb habe der Landesherr durch andere Mittel versucht, Einfluss auf die Jagd zu nehmen, indem er sich beispielsweise die Vorjagd sicherte, $d$. h., er nahm sich das Recht heraus, am Beginn der Jagdsaison exklusiv das Revier des Jagdrechtinhabers zu bejagen, wo er noch den besten Wildbestand vorfand. ${ }^{131}$

Weitere ähnliche Einschränkungen konnten die Mitjagd (der Landesherr war dann parallel zum Besitzer des Grundes jagdberechtigt), die stille Jagd (nur mit Fallen) oder die Klapperjagd (das Wild wurde nur durch Lärm aufgescheucht) sein. Diese jagdrechtlichen Kompromisse und komplizierten Abstufungen konnten nur dort entstehen, wo eine stark ausgeprägte Konkurrenz zwischen Landesherr und Adel stattfand wie eben in Bayern aufgrund der zumindest um 1500 sehr starken Position des Adels vor allem im Landtag. ${ }^{132}$

\section{Das Jagdrecht in Tirol}

Maximilian I. war ein begeisterter Jäger, und dementsprechend war auch das Jagdrecht unter seiner Herrschaft: Die Strafen, die im Fall von unerlaubtem Töten des Wildes verhängt wurden, waren streng. Mit diesem Jagdrecht wurde auch eine starke Hege des Wildes betrieben, indem Adelige und auch Klöster, die noch das Jagdrecht und ein Revier hatten, Personal einstellen mussten, das für das Überwachen und die Hege des Wildbestandes zuständig war. ${ }^{133}$

Unter Maximilian leistete sich Tirol einen großen Personalstand, der nur für Jagdangelegenheiten zuständig war. Heinrich Oberrauch hat dies in seinem Werk eindrücklich herausgearbeitet. Dieser große Jägerbedarf hatte auch damit zu tun, dass Tirol als sehr wildreiches Gebiet galt. Allein um die Falkenbeiz auszuüben, hatte Maximilian 15 Falkenmeister und 60 Falkenknechte am Hof in Innsbruck. Eine Aufgabe der vom Kaiser angestellten Jäger war es, den Wildbestand zu überwachen und dafür zu sorgen, dass keine unerlaubten Abschüsse, vor allem an Hirschen und Gämsen, geschahen. ${ }^{134}$

128 Franz, Die Landesordnung von 1516/1520, S. 115

129 Ebd., S. 23

130 Eckardt, Herrschaftliche Jagd, bäuerliche Not und bürgerliche Kritik, S. 39

131 Ebd., S. 40. Siehe auch: Rösener, Geschichte der Jagd, S. 217.

132 Eckardt, Herrschaftliche Jagd, bäuerliche Not und bürgerliche Kritik, S. 40 f. Siehe auch: Rösener, Geschichte der Jagd, S. 217.

133 Nussbaumer, Es begann mit der Jagd, S. 97 f.

134 Oberrauch, Tirols Wald und Waidwerk, S. 53-94. 
Die Jagd auf das hohe Wild, also vornehmlich Rotwild und Gämse, wurde unter Maximilian generell verboten mit der Ausnahme, dass nachgewiesen werden konnte, dass das Wild zu großen Schaden angerichtet hatte. ${ }^{135}$

Die starken Einschränkungen führten auch zu Widerstand. Zunächst vor allem von den Bauern, die von Schäden durch überhegtes Wild betroffen waren. Daneben verärgerten auch die Verbote, Zäune anzulegen und Hunde frei laufen zu lassen. Diese Faktoren führten zu den so genannten Wildereraufständen in Tirol, die meist dann stattfanden, wenn die Nachricht vom Tod des Landesherrn eintraf. So geschehen nach dem Tod Maximilians 1519, Ferdinand Karl 1663 und Sigismund Franz 1666. Interessant daran ist jedoch nicht nur der Widerstand der Bauern, sondern dass sich offensichtlich auch der niedere Adel daran beteiligte, der mit den Einschränkungen des Jagdrechtes nicht einverstanden war. ${ }^{136}$

Als Reaktion auf die Wilderei nach dem Tod Maximilians wurde das Jagdrecht unter dessen Nachfolger Ferdinand I. verschärft. Er nahm alle Tiere außer Schneehasen und Schneehühner für sich in Anspruch. Diese Bestimmungen wurden im Bauernkrieg stark gelockert und danach, als Ferdinand wieder in besserer Position war, wieder eingeschränkt. Jagd auf Raubwild blieb aber in eingeschränkter Weise erlaubt. ${ }^{137}$

Da es in Tirol sehr viele freie Bauern gab, war es schwierig, ihnen die Jagdausübung generell zu verbieten, daher durften in Tirol steuerzahlende Untertanen und auch Bergwerksbedienstete zumindest die niedere Jagd ausführen, wobei in diesem Fall nur Hasen, Füchse und Rebhühner gemeint waren, wobei zusätzlich nur das zumeist sehr kleine Gebiet des "Hofzaun“ dafür vorgesehen war. Die hohe Jagd blieb beim Adel, vornehmlich beim Landesherrn. ${ }^{138}$

Der niedere Adel hatte zur Jagd maßgeblich Zugang durch die sehr häufig vom Landesherrn angestellten Lustjagden, teils mehrere Hundert Adelige nahmen daran teil, und dementsprechend ausgiebig wurde gejagt. Hunderte Gämsen und Steinböcke dürften bei solchen Jagden getötet worden sein. ${ }^{139}$

Angesichts der Schwierigkeit, den freien Untertanen die Jagd auf kleine Tiere sowie Raubwild zu verbieten, und der starken Einschränkung der restlichen Jagd vor allem für den niederen Adel, zeigt sich ein sehr differenziertes Bild für Tirol. Der landsässige Adel hatte zur Jagd hauptsächlich nur bei Lustjagden des Landesherrn Zugang, woraus sich wahrscheinlich auch die Teilnahme an Widerstandsaktionen durch massenhaftes Abschießen von Wild nach dessen Tod erklären lässt.

135 Nussbaumer, Es begann mit der Jagd, S. 98.

136 Ebd., S. 98-100.

137 Franz Niederwolfsgruber, Kaiser Maximilians I. Jagd- und Fischereibücher. Jagd und Fischerei in den Alpenländern im 16. Jahrhundert, Innsbruck 1965, S. 46-48.

138 Ebd., S. 46-48. Zu den Rechten eines Freien in Tirol siehe auch: Oberrauch, Tirols Wald und Waidwerk, S. $14 \mathrm{f}$.

139 Oberrauch, Tirols Wald und Waidwerk, S. 83. 
Noch Maria Theresia beschwerte sich 1740 über die Tiroler Untertanen, die die Jagd als rechtmäßig anerkannten, wenn der Landesherr starb. ${ }^{140}$ Dabei ging die Bevölkerung häufig davon aus, dass das Jagdrecht an die Person des Landesherrn gebunden war und mit dessen Tod erlosch. ${ }^{141}$ Schennach arbeitet hier die Wilderei nach dem Tod der Landesherrn 1519 und 1619 heraus und kommt dabei zu einem sehr differenzierten Bild, das hier nur in aller Kürze wiedergegeben werden kann: Wie stark die Wilderei nach dem Tod des Landesherrn war, hing zumeist davon ab, wie streng dessen Jagdrecht ausgelegt war und wie sehr einerseits die Bauern von Wildschäden betroffen waren und andererseits, wie stark der Zugang des niederen Adel zur Jagd war. ${ }^{142}$

Ursprünglich hatte der Adel in Tirol bei Verleihung oder Verpfändung von Gerichtsherrschaften das Jagdrecht mit erhalten, was sich erst mit Maximilian I. änderte, indem Jagdrechte bei der Vergabe dem Landesfürsten vorbehalten blieben. ${ }^{143}$ Daher kam es auch regelmäßig zu Auseinandersetzungen zwischen Adel und Regierung über die Existenz und den Umfang der Jagdrechte. Beispiele hierfür sind die Auseinandersetzungen mit den Familien Schrofenstein und Liechtenstein. ${ }^{144}$

Zu einem, wie Schennach formuliert, „Proteststurm“ des Adels und auch des Klerus führte das bereits erwähnte Mandat Ferdinands I., indem er alle Tiere außer Schneehase und Schneehuhn für sich in Anspruch nahm. In der Folge wurde erklärt, "dass Adel und Prälaten die Jagd auf Fuchs, Hasen und schädliche Tiere (wie Bären oder Wölfe) weiterhin zustehen sollte."145 Gleiches galt für Vögel, nachdem Ferdinand eingestand, die Rechte des Adels verletzt zu haben. ${ }^{146}$ Ein zusätzlicher Weg, wie der Adel an die Jagd kommen konnte, war die Gewährung von „Gnadenjagden“. Der Adelige musste für sie ansuchen und bekam anschließend, wenn er es sich verdient hatte, eine bestimmte Anzahl an (Hoch-)Wild zugestanden. ${ }^{147}$

\section{Vergleich}

Ein auffallender Punkt im Jagdrecht ist folgender: Je näher der Landesherr residierte oder je öfter er in das betreffende Gebiet kam, desto strenger war das Jagdrecht zu Lasten der Untertanen, auch des niederen Adels, eingeschränkt und desto mehr behielt sich der Landesherr vor. Ein gutes Beispiel hierfür sind die restriktiven Einschränkungen Kaiser Maximilians und seines Nachfolgers Ferdinand in Tirol, was auch mit der schwächeren Position des Adels in Tirol im Vergleich zu Bayern begründet werden kann. In Bayern hatte der Herzog viel größere Schwierigkeiten, sein Recht durchzusetzen, und sobald der Landesherr keinen Hof mehr in Tirol hielt, war auch hier die Durchsetzung des bestehenden Rechtes schwierig geworden.

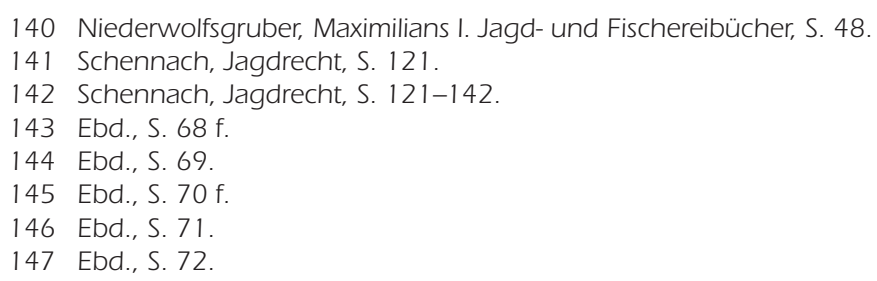




\section{Schluss}

Abschließend sollen die Ergebnisse kurz zusammengefasst und kommentiert werden. Weiters soll in diesem Abschnitt überprüft werden, ob auf die formulierte These und die Fragestellungen eine Antwort, eventuell eine Bestätigung gefunden werden konnte, wobei folgendes Zitat als Ausgangspunkt hilfreich ist:

„Der Adel wird aus der Rolle von Gefolgsleuten des Herzogs in diejenige von Untertanen verdrängt, eine Entwicklung, die sich im 15. Jahrhundert schon anbahnt, als eine naturalistischere Betrachtungsweise des Staates zum Tragen kommt, die ja auch in einer tiefgreifenden Veränderung des Fürstentypus' ihren Ausdruck findet: Albrecht IV. von Bayern (1465-1508) ist ein rechnender Fürst, der sein Land von der Schreibstube, nicht mehr vom Sattel aus regiert. Im 16. Jahrhundert sieht sich der Adel obendrein in zunehmenden Maße aus dem fürstlichen Hofgericht verdrängt, als der um sich greifende Gebrauch des römischen Rechts die Anwesenheit geschulter Juristen erforderlich macht." ${ }^{148}$

Dieser Einschätzung Andrian-Werburgs ist zuzustimmen. Im 16. Jahrhundert veränderten sich die Vorzeichen, unter denen regiert wurde. Durch zunehmende Institutionalisierung und Rationalisierung wurde der Adel immer mehr aus dem Herrschaftsbereich hinausgedrängt. Das zeigt sich besonders deutlich in der Gerichtsbarkeit: Nicht nur, dass der landsässige Adel sowohl in Bayern als auch in Tirol die Hochgerichtsbarkeit vorwiegend verlor, auch die Niedergerichtsbarkeit wurde zunehmend eingeschränkt. Die Verbreitung des römischen Rechtes als Grundlage der Jurisdiktion verlangte nach ausgebildeten Spezialisten, was der Adel in der Regel nicht war. Kamen Adelige erneut in den „Genuss" der Ausübung der Gerichtsbarkeit, war dies zumindest in Tirol Ergebnis der permanenten Geldsorgen des Landesherren.

In der Jagd zeigt sich dies in weniger starker Form, auch wenn diese mit der Gerichtsbarkeit stark zusammenhängt. Dennoch kam es hier zu Konflikten des niederen Adels mit den Landesherrn, wie etwa die Beteiligung Adeliger an den Wildereraufständen des 16. Jahrhunderts in Tirol zeigt. Die Jagd war wichtiger Bestandteil der adeligen Kultur und Standesauffassung, von der sich der Adel nur ungern trennte, was schon beim Landesherren begann. Musste der Landesherr beispielsweise während der Bauernkriege die niedere Jagd freigeben, war dies eine der ersten Maßnahmen, die er wieder rückgängig machte, sobald sich seine Position gebessert hatte. Die Jagdprivilegien gab der Adel in der Folge erst in der Mitte des 19. Jahrhunderts ab.

Auch das Vorrecht, keine Steuern zahlen zu müssen, war nicht in der Weise von einem Rückgang betroffen, wie dies bei der Gerichtsbarkeit gesehen werden konnte. Zwar gibt es einige bedeutende Ausnahmen vom Prinzip der Steuerfreiheit des Adels, doch in der Regel blieb der Adel weitgehend steuerfrei. Die Steuern, die er bezahlen musste, waren von bescheidenem Umfang. Die Konflikte, die gegenüber anderen Schichten entstanden, betrafen daher vermehrt prinzipielle Fragen.

148 Andrian-Werburg, Der altbaierische Adel, S. 56. 
Dennoch war der Adel in keiner Weise homogen, wie die Betrachtungen der einzelnen Privilegien in den Territorien Bayern und Tirol gezeigt hat. Es gab nicht nur Konflikte wegen (Vor-)Rechten nach oben oder unten, sondern auch innerhalb des Adels. So wehrte sich der Adel gegen „Eindringlinge“, wohlhabende Bürger, die durch Nobilitierung adelig geworden waren. In Bayern ist hier die starke Betonung des Edelmannsfreien zu bemerken. Mit diesem Status waren zusätzliche Rechte verbunden, wie etwa die Niedergerichtsbarkeit, und damit einhergehend auch Jagdrechte, die einen besseren niederen Adel anzeigen sollten. Die Edelmannsfreiheit zu erlangen war dabei schwierig und verlangte nach Nachweisen. Das zeigt, dass der Brief- bzw. Neuadel innerhalb des Adels schlecht angesehen war und eine nochmalige Abschottung innerhalb des eigenen Standes stattfand, um denselben elitär zu halten. ${ }^{149}$

Die Heterogenität des Adels hebt auch Bieberstein hervor, der diesen als „in sich vielfältig gegliedert" beschreibt, ${ }^{150}$ und auch Johanek erkennt, dass es schwierig ist, ein Bild des Adels in seinen Untergruppen und einzelnen Gliedern abzubilden und zu zeigen, wie „einzelne Geschlechter ihren Status verteidigen, wahren können oder ihn verlieren. "151

Die Institutionalisierung, das "Staatwerden", die zunehmende Verwaltung und der Aufstieg des Beamtentums waren für den Adel in dieser Zeit schwere Belastungen, mit denen er kaum mithalten konnte bzw. deren Durchsetzung seine Privilegien in Frage stellte und denen er sich anpassen musste. Hier fällt auch die Schaffung des Reichskammergerichtes hinein - eine Institution, deren Gründungsdatum häufig zur Markierung der Epochengrenze von Mittelalter und Neuzeit herhalten muss ${ }^{152}$ - mit der auch das Verbot der Fehde einherging: Die Fehde war ursprünglich die Rechtslösung im Streit zwischen zwei Adeligen. Neben der Herausbildung von Spezialisten als Richter war auch die Möglichkeit für Adelige eingeschränkt worden, Streitigkeiten „auf ihre Art und Weise" zu lösen. Der Adel musste sich dementsprechend eingeschränkt in seiner Wirkungskraft fühlen und war genötigt, seine Privilegien in andere Richtungen auszubauen. Allgemein ist das 16. Jahrhundert daher von einem leichten Rückgang der adeligen Vorrechte geprägt, was durch den wachsenden Staatsapparat beschleunigt worden sein dürfte. Daher ist es erstaunlich, dass der Adel noch so lange an seinen Rechten festhalten und seine Stellung großteils bewahren konnte.

Abschließend bleibt festzuhalten: Weder stand jedes der untersuchten Privilegien ausschließlich dem Adel zu, noch stand jedes immer dem Adel zu. Steuerfreiheit genoss nicht nur der Adel, sondern auch bestimmte Berufs- und Personengruppen. Der Adel war zudem nicht gänzlich von Steuern befreit. Die Gerichtsbarkeit war in der Frühen Neuzeit nicht mehr ausschließlich in der Hand des Adels, und auch nicht jeder Adelige durfte aufgrund seines Standes Recht sprechen. Das Jagdrecht war eines der Felder, die in der Frühen Neuzeit verstärkt in den alleinigen Bereich des Landesherren kamen.

149 Vgl. dazu: Endres, Adel in der Frühen Neuzeit, S. 34.

150 Bieberstein, Adelsherrschaft und Adelskultur, S. 3.

151 Johanek, Adel in den österreichischen Ländern und in Tirol, S. 15

152 Wieland, Die Ausnahme in der Sprache des Allgemeinen, S. 120. 


\section{Literatur und Quellen}

Andrian-Werburg, Klaus Freiherr von, Der altbaierische Adel im landesfürstlichen Staat der Wittelsbacher bis zum Abschluss der ritterschaftlichen Verfassung, in: Rössler, Helmuth (Hrsg.), Deutscher Adel. 1430-1555. Büdinger Vorträge 1963 (Schriften zur Problematik der deutschen Führungsschichten in der Neuzeit 1), Darmstadt 1965, S. 48-57.

Bavarikon, Die erklärte Landesfreiheit des Herzogtums Bayern von 1508, [http://bavarikon. de/de/bookviewer/kpbO-GDA-OBJ-0000000000000024_00001],2013, eingesehen 19.2.2014.

Bavarikon, Der 60. Freiheitsbrief der bayerischen Landstände: Verleihung der Edelmannsfreiheit, [http://bavarikon.de/de/image/kpbO-GDA-OBJ-0000000000000 026], 2013, eingesehen 19.2.2014.

Bieberstein, Johannes Rogalla von, Adelsherrschaft und Adelskultur in Deutschland (Aus dem Deutschen Adelsarchiv 14), Limburg ${ }^{3} 1998$.

Bleeck, Klaus/Garber, Jörn, Nobilitas: Standes- und Privilegienlegitimation in deutschen Adelstheorien des 16. und 17. Jahrhunderts, in: Daphnis 11 (1982), H. 1-2, S. 49-114.

Bonazza, Marcello, La nobiltà trentino-tirolese di fronte al prelievo fiscale tra politica di ceto e comporrtamenti individuali, in: Geschichte und Region 4 (1995), S. 77-112.

Buszello, Horst, Legitimation, Verlaufsformen und Ziele, in: Blickle, Peter/Buszello, Horst/ Endres, Rudolf (Hrsg.), Der deutsche Bauernkrieg, Paderborn u. a. ${ }^{3} 1995$, S. 281-321.

Conze, Eckart, Adel, in: ders. (Hrsg.), Kleines Lexikon des Adels. Titel, Throne, Traditionen, München 2005, S. 15-18.

Demel, Walter, Der europäische Adel. Vom Mittelalter bis zur Gegenwart, München 2005.

Eckardt, Hans Wilhelm, Herrschaftliche Jagd, bäuerliche Not und bürgerliche Kritik. Zur Geschichte der fürstlichen und adligen Jagdprivilegien vornehmlich im südwestdeutschen Raum (Veröffentlichungen des Max-Planck-Instituts für Geschichte 48), Göttingen 1976.

Endres, Rudolf, Adel in der Frühen Neuzeit (Enzyklopädie Deutscher Geschichte 18), München 1993.

Ernst, Viktor, Die Entstehung des niederen Adels, Berlin 1916.

Estienne, Charles/Jean Liébault, XV. Bücher von dem Feldbaw vnd recht volkommener Wolbestellung eines bekömmlichen Landsitzes / vnnd geschicklich angeordneten Meyerhofs oder Landguts / Sampt allem / was demselben Nutzes vnd Lusts halben anhängig, Straßburg 1598.

Franz, Monika Ruth, Die Landesordnung von 1516/1520. Landesherrliche Gesetzgebung im Herzogtum Bayern in der ersten Hälfte des 16. Jahrhunderts (Bayerische Rechtsquellen 5), München 2003.

Gaismair, Michael, Landesordnung für Tirol (1526). Einleitung von Nils Petersen, in: Strohm, Theodor/Klein, Michael (Hrsg.), Die Entstehung einer sozialen Ordnung Europas. 
Historische Studien und exemplarische Beiträge zur Sozialreform im 16. Jahrhundert (Veröffentlichungen des Diakoniewissenschaftlichen Instituts an der Universität Heidelberg 22), Heidelberg 2004, S. 266-276.

Heydenreuter, Reinhard, Strafrechtspflege in den bayerischen Besitzungen des Hochstifts Freising, in: Glaser, Hubert (Hrsg.), Hochstift Freising. Beiträge zur Besitzgeschichte, München 1990, S. 217-228.

Heydenreuter, Reinhard, Zur Rechtstellung des landsässigen Adels im Kurfürstentum Bayern zwischen dem 16. und 18. Jahrhundert, in: Demel, Walter/Kramer, Ferdinand (Hrsg.), Adel und Adelskultur in Bayern (Zeitschrift für bayerische Landesgeschichte Beiheft 32), München 2008, S. 43-105.

Johanek, Peter, Der Adel in den österreichischen Ländern und in Tirol während des späten Mittelalters und der frühen Neuzeit, in: Andermann, Kurt/Pfeifer, Gustav (Hrsg.), Die Wolkensteiner. Facetten des Tiroler Adels in Spätmittelalter und Neuzeit (Veröffentlichungen des Südtiroler Landesarchivs 30), Innsbruck 2009, S. 11-28.

Kink, Barbara, Adelige Lebenswelt in Bayern im 18. Jahrhundert. Die Tage- und Ausgabebücher des Freiherrn Sebastian von Pemler von Hurlach und Leutstetten (17181772) (Studien zur bayerischen Verfassungs- und Sozialgeschichte 26), München 2007.

Kopfmann, Klaus, Wirtschaftliche Grundlagen - die Hofmarken, in: Jahn, Wolfgang/ Hamm, Margot/Brockhoff, Evamaria (Hrsg.), Adel in Bayern. Ritter, Grafen, Industriebarone, Stuttgart 2008, S. 104-105.

Köfler, Werner, Land - Landschaft - Landtag. Geschichte der Tiroler Landtage von Anfängen bis 1808 (Veröffentlichungen des Tiroler Landesarchivs 3), Innsbruck 1985.

Körner, Martin, Steuern und Abgaben in Theorie und Praxis im Mittelalter und in der frühen Neuzeit, in: Schremmer, Eckart (Hrsg.), Steuern, Abgaben und Dienste vom Mittelalter bis zur Gegenwart. Referate der 15. Arbeitstagung der Gesellschaft für Sozialund Wirtschaftsgeschichte vom 14. bis 17. April 1993 in Bamberg Nierteljahresschrift für Sozial - und Wirtschaftsgeschichte Beiheft 114), Stuttgart 1994, S. 53-76.

Ksoll, Margit, Diewirtschaftlichen Verhältnisse des bayerischenAdels 1600-1679. Dargestellt an den Familien Törring-Jettenbach, Törring zum Stain sowie Haslang zu Haslangkriet und Haslang zu Hohenkammer (Schriftenreihe zur bayerischen Landesgeschichte 83), München 1986.

Kummer, Katrin Ellen, Landstände und Landschaftsverordnung unter Maximilian I. von Bayern (1598 - 1651) (Schriften zur Verfassungsgeschichte 74), Berlin 2005.

Niederwolfsgruber, Franz, Kaiser Maximilians I. Jagd- und Fischereibücher. Jagd und Fischerei in den Alpenländern im 16. Jahrhundert, Innsbruck 1965.

Nussbaumer, Johann, Es begann mit der Jagd, Wien 1984.

Oberrauch, Heinrich, Tirols Wald und Waidwerk. Ein Beitrag zur Forst- und Jagdgeschichte (Schlern-Schriften 88), Innsbruck 1952. 
Palme, Rudolf, Frühe Neuzeit. (1490-1665), in: Fontana, Josef u.a. (Hrsg.), Geschichte des Landes Tirol, Bd. 2, Bozen 1986.

Painer, Barbara, Geschichte des Steuerwesens der Stadt Meran von 1438-1780, Diss., Innsbruck o.J.

Pelzer, Erich, Der elsässische Adel im Spätfeudalismus. Tradition und Wandel einer regionalen Elite zwischen dem Westfälischen Frieden und der Revolution (1648-1790) (Ancien Régime, Aufklärung und Revolution 21), München 1990.

Rösener, Werner, Die Geschichte der Jagd. Kultur, Gesellschaft und Jagdwesen im Wandel der Zeit, Düsseldorf-Zürich 2004.

Schennach, Martin P., Jagdrecht, Wilderei und "gute Policey". Normen und ihre Durchsetzung im frühneuzeitlichen Tirol (Studien zu Policey und Policeywissenschaft), Frankfurt a. M. 2007.

Schennach, Martin P., Gesetz und Herrschaft. Die Entstehung des Gesetzgebungsstaates am Beispiel Tirols (Forschungen zur deutschen Rechtsgeschichte 28), Köln-Weimar-Wien 2010.

Schlachta, Astrid von, Das Amt des Landeshauptmanns - Verwaltung und Politik in Tirol im 18. Jahrhundert am Beispiel Paris Dominkus von Wolkenstein-Trostburgs und Paris von Wolkenstein-Rodeneggs, in: Andermann, Kurt/Pfeifer, Gustav (Hrsg.), Die Wolkensteiner. Facetten des Tiroler Adels in Spätmittelalter und Neuzeit (Veröffentlichungen des Südtiroler Landesarchivs 30), Innsbruck 2009, S. 345-359.

Schneider, Joachim, Spätmittelalterlicher deutscher Niederadel. Ein landschaftlicher Vergleich (Monographien zur Geschichte des Mittelalters 52), Stuttgart 2003.

Schomburg, Walter, Lexikon der deutschen Steuer- und Zollgeschichte. Abgaben, Dienste, Gebühren, Steuern und Zölle von den Anfängen bis 1806, München 1992.

Schuster, Wolfgang, o.T. in: Wolfgang Jahn/Margot Hamm/Evamaria Brockhoff (Hrsg.), Adel in Bayern. Ritter, Grafen, Industriebarone, Stuttgart 2008, S. 111.

Sikora, Michael, Der Adel in der Frühen Neuzeit (Geschichte kompakt), Darmstadt 2009.

Stolz, Otto, Politisch-historische Landesbeschreibung von Südtirol. Zweiter Teil der Landesbeschreibung von Tirol (Schlern-Schriften 40), Innsbruck 1937.

Stone, Lawrence, The Crisis of the Aristocracy: 1558-1641, Oxford 1965.

Strachwitz, Moritz, Niederer Adel, in: Conze, Eckart (Hrsg.), Kleines Lexikon des Adels. Titel, Throne, Traditionen, München 2005, S. 187.

Stutzer, Dietmar, Zwei Hofmarken im Vergleich: Stachesried und Hilling, in: Jahn, Wolfgang/Hamm, Margot/Brockhoff, Evamaria (Hrsg.), Adel in Bayern. Ritter, Grafen, Industriebarone, Stuttgart 2008, S. 105-117.

Wenzl, Stephanie von, Stadt und Adel in Bruneck. Auseinandersetzungen in der 2. Hälfte des 16. Jahrhunderts, Innsbruck Dipl. 2006. 
Wieland, Christian, Die Ausnahme in der Sprache des Allgemeinen. Bayerischer Adel und Gericht im 16. Jahrhundert, in: Demel, Walter / Kramer, Ferdinand (Hrsg.), Adel und Adelskultur in Bayern (Zeitschrift für bayerische Landesgeschichte Beiheft 32), München 2008, S. 107-135.

Nikolaus Bliem ist Student des Masterstudiums Geschichte im 2. Semester und studentischer Mitarbeiter am Institut für Geschichtswissenschaften und Europäische Ethnologie der Universität Innsbruck. nikolaus.bliem@student.uibk.ac.at

\section{Zitation dieses Beitrages}

Nikolaus Bliem, Die Vorrechte des Adels in Bayern und Tirol. Steuer-, Gerichts- und Jagdprivileg im 16. und 17. Jahrhundert, in: historia.scribere 7 (2015), S. 297-330, [http://historia.scribere.at], 2014-2015, eingesehen 1.3.2015 (=aktuelles Datum).

(C) Creative Commons Licences 3.0 Österreich unter Wahrung der Urheberrechte der Autorlnnen. 
